\title{
Rheo-Optical Studies on the Deformation Mechanism of Semicrystalline Polymers. XIII. Uniaxial Deformation Mechanism of Polyethylene Spherulites as Observed by Orientation Distribution Function of Crystallites ${ }^{\dagger}$
}

\author{
Ken-ichi Fujita, * Shoji Suehiro, Shunji Nomura, ${ }^{* *}$ \\ and Hiromichi KAWAI*** \\ Department of Polymer Chemistry, Faculty of Engineering, \\ Kyoto University, Kyoto 606, Japan.
}

(Received February 13, 1982)

\begin{abstract}
The crystal orientation behavior during stretching of a spherulitic high-density polyethylene was observed from the standpoint of changes in uniaxial orientation distribution functions $q_{j}\left(\zeta_{j}, 0\right)$ for 13 different $(j$-th) crystal planes and further $w(\xi, 0, \eta)$ for crystallites (crystal grains) both as functions of the extension ratio of a bulk specimen. It was found that two populations develop in $w(\xi, 0, \eta)$ at $\theta$ somewhat larger than $30^{\circ}$ and $\eta$ of $90^{\circ}$ and at $\theta$ a little smaller than $30^{\circ}$ and $\eta$ of $0^{\circ}$ and move to smaller values of $\theta$ to some extent, as the extension ratio was increased up to 1.4. The former population seemed to develop posteriorly but at a much greater rate than the latter population. The uniaxial deformation mechanism of polyethylene spherulites is discussed quantitatively by comparing the dependence of $w(\xi, 0, \eta)$ upon the extension ratio with that calculated from a spherulite deformation model which combines the crystal lamellar orientation in affine fashion with the reorientation of the crystal grains within the orienting lamellae. The crystal reorientation mechanism may be represented by two preferential fashions which involve rotations of the crystal grains about their own $a$ - or $b$-axes so as to orient their $c$-axes toward the stretching direction. The rotations of the crystal grains about the crystal $a$ - or $b$-axes must be associated, respectively, with intra-lamellar shearing or lamellar detwisting and must be accentuated in the polar or equatorial zone of the uniaxially deformed spherulites to give the population at $\theta$ a little larger than $30^{\circ}$ and $\eta$ of $90^{\circ}$ or at $\theta$ a little smaller than $30^{\circ}$ and $\eta$ of $0^{\circ}$.

KEY WORDS Rheo-Optical Properties / High-Density Polyethylene / Deformation Mechanism of Spherulites / Orientation Distribution Function of Crystal Grains / Microparacrystallite Domain / Crystal Mosaic Block / Inter- and Intra-Lamellar Grain Boundary Phenomena /
\end{abstract}

The deformation mechanism of semicrystalline polymers has been extensively studied by a number of authors using different optical techniques, such as polarized microscopy, electron microscopy, small-angle polarized light scattering, and smallangle X-ray scattering, in order to investigate the mechanism in terms of the change in crystalline texture during deformation. These investigations were carried out primarily for polyethylene specimens; ${ }^{1-25}$ which included not only bulk-crystallized specimens having spherulitic and row-nucleated crystalline textures, but also solution-grown specimens of single crystal as well as of spherulitic texture in two-dimension of ultrathin thickness.

Some of the investigations were performed for a bulk-crystallized specimen of spherulitic texture by

${ }^{\dagger}$ Presented at the Symposium on Fiber Science \& Technology, the Society of Fiber Science \& Technology, Japan, October 16-17, 1979, Nagoya, Japan.

* On leave from the Department of Polymer Engineering, Nagoya Institute of Technology, Gokisho, Showa-ku, Nagoya 466, Japan.

** Present address: Department of Textile Engineering, Kyoto University of Industrial Arts \& Fiber Technology, Matsugasaki, Sakyo-ku, Kyoto 606, Japan.

*** To whom correspondence should be addressed. 
comparing the orientation behavior of crystallites within the texture during the deformation of the specimen, as observed by X-ray diffraction, with those predicted from a deformation model for the spherulitic texture, in hope of characterizing the mechanism in terms of the model parameters. Earlier quantitative investigations were concerned with a comparison of the second-order orientation factors for particular crystallographic axes of the crystallites. ${ }^{4,5,7,8,10}$ However, these factors are nothing but averages (the second moments) of the orientation distributions of the axes, and not necessarily sufficient to deduce detailed information on the deformation mechanism of the spherulitic texture. This shortcoming has been greatly eliminated by Nomura et al. ${ }^{18,19}$, who used the orientation distribution functions of the particular crystallographic axes. A further improvement was to use the orientation distribution function of the crystallites themselves, as preliminarily reported by Matsuo et al. ${ }^{23}$

In the previous papers of this series of rheooptical studies on the deformation mechanism of semi-crystalline polymers, ${ }^{26-33}$ the dynamic mechanical dispersions of polyethylene have been intensively investigated for bulk-crystallized specimens of spherulitic as well as row-nucleated crystalline textures, using dynamic $\mathrm{X}$-ray diffraction ${ }^{34}$ and dynamic birefringence ${ }^{35}$ techniques. As have been reviewed for high-density polyethylenes with spherulitic and row-nucleated crystalline textures ${ }^{36}$ and for low- to high-density polyethylenes all having spherulitic crystalline texture, ${ }^{37}$ the $\alpha\left(=\alpha_{1}\right)$ and $\beta$ mechanical dispersions of polyethylene have been assigned to intra- and inter-lamellar relaxation phenomena, respectively, both being accompanied with dynamic deformation of the crystalline textures.

In this paper, we first discuss the uniaxial deformation mechanism of polyethylene spherulites again quantitatively. This involves a comparison of the orientation distribution functions of the crystal grains $w(\xi, 0, \eta)$, as observed by X-ray diffraction of a bulk specimen stretched to various extension ratios, with those predicted from a model for spherulite deformation, as proposed by Oda, Nomura, and $\mathrm{Kawai}^{7}$ and further by Nomura et al. ${ }^{18,19}$ With the objective of elucidating the deformation mechanism in terms of the model parameters in more detail, we will extend our discussion in forthcoming papers to include the dynamic deformation mechanism of the crystalline texture by investigating the dynamic response of the orientation distribution function of the crystal grains $\Delta w^{*}(\xi, 0, \eta)$ so as to clarify the $\alpha$ and $\beta$ mechanical dispersions of polyethylene more definitely.

\section{INVESTIGATION OF ORIENTATION DISTRIBUTION FUNCTION OF CRYSTALLITES}

Figure 1(a) shows the orientation of a Cartesian

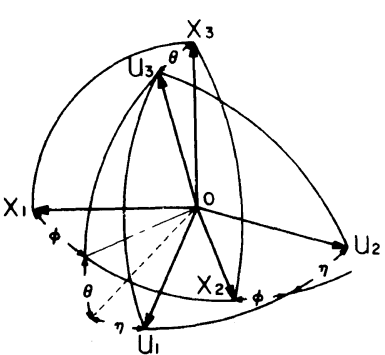

(a)

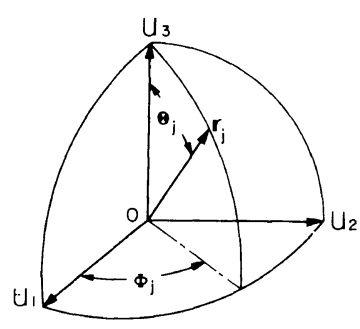

(b)

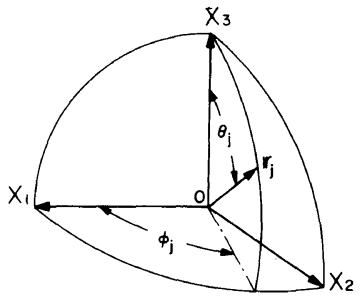

(c)

Figure 1. (a) three Euler angles, $\phi, \theta$, and $\eta$, specifying the orientation of a Cartesian coordinate system of $0-u_{1} u_{2} u_{3}$ fixed within a crystallite with respect to another Cartesian coordinate system of $0-x_{1} x_{2} x_{3}$ fixed within bulk specimen: (b) polar and azimuthal angles, $\Theta_{j}$ and $\Phi_{j}$, specifying the orientation of reciprocal lattice vector of the $j$-th crystal plane, $r_{j}$ with respect to the Cartesian coordinate system of $0-u_{1} u_{2} u_{3}$ fixed within the crystallite in such that the $u_{1}, u_{2}$, and $u_{3}$ axes are parallel to the crystal $a$-, $b$-, and $c$-axes of polyethylene orthorhombic crystal, respectively: and (c) polar and azimuthal angles, $\theta_{j}$ and $\phi_{j}$, specifying the orientation of the $r_{j}$ vector with respect to the Cartesian coordinate system of $0-x_{1} x_{2} x_{3}$ fixed within the bulk specimen in such a way that the $x_{3}, x_{2}$, and $x_{1}$ axes are parallel to the machine, transverse, and thickness directions of the bulk specimen, respectively. 
coordinate system $0-u_{1} u_{2} u_{3}$ fixed within a crystallite (or crystal grain) relative to another Cartesian coordinate system $0-x_{1} x_{2} x_{3}$ fixed within the bulk specimen in terms of three Euler angles of $\phi, \theta$, and $\eta$. The Cartesian coordinate $0-u_{1} u_{2} u_{3}$ must be fixed within the polyethylene crystal (orthorhombic crystal) in such a way that the $u_{1}, u_{2}$, and $u_{3}$ are parallel to the crystal $a-, b$-, and $c$-axis, respectively, so as to make the properties of this coordinate system orthorhombically symmetric. The Cartesian coordinate $0-x_{1} x_{2} x_{3}$ must also be fixed within the bulk specimen so as to make any orientation distribution of the crystallites, symmetric with respect to the coordinate axes as high as possible; i.e., the $x_{3}, x_{2}$, and $x_{1}$ axes are parallel to the machine, transverse, and thickness directions, respectively, for a sheetlike specimen stretched orthogonal-biaxially or uniaxially. These fixations of the coordinate axes taking into account the symmetries, as mentioned above, are essential for making a mathematical description of the orientation of the crystallites within the bulk specimen simpler, as will be shown later. Figures 1(b) and 1(c) show the orientation of a given vector $r_{j}$ (reciprocal lattice vector of the $j$-th crystal plane) within the crystallite and the bulk specimen, respectively, in terms of the polar and azimuthal angles $\Theta_{j}$ and $\Phi_{j}$ and $\theta_{j}$ and $\phi_{j}$.

The two sets of angles, $\left(\theta_{j}, \phi_{j}\right)$ and $\left(\Theta_{j}, \Phi_{j}\right)$ referring to the identical vector $r_{j}$, are related to each other through a linear transformation of the two Cartesian coordinate systems accompanied by the rotations of the coordinate axes by $\phi, \theta$, and $\eta$, and the interrelation between the seven angles defined can be given by

$$
\left(\begin{array}{l}
\sin \theta_{j} \cos \phi_{j} \\
\sin \theta_{j} \sin \phi_{j} \\
\cos \theta_{j}
\end{array}\right)=\left(t_{\mathrm{ki}}\right)\left(\begin{array}{l}
\sin \Theta_{j} \cos \Phi_{j} \\
\sin \Theta_{j} \sin \Phi_{j} \\
\cos \Theta_{j}
\end{array}\right)
$$

where $\left(t_{k i}\right)$ is a linear transformation operator (the direction cosine of each of $u_{k}$ axes with respect to each of $x_{i}$ axes).

The orientation distribution functions of the crystallites and the $r_{j}$ vectors within the bulk specimen, $w(\theta, \phi, \eta)$ and $q_{j}\left(\theta_{j}, \phi_{j}\right)$, are subjected, respectively, to the following normalization conditions;

$$
\int_{\eta=0}^{2 \pi} \int_{\phi=0}^{2 \pi} \int_{\xi=-1}^{1} w(\xi, \phi, \eta) \mathrm{d} \xi \mathrm{d} \phi \mathrm{d} \eta=1
$$

$$
\int_{\phi_{j}=0}^{2 \pi} \int_{\zeta_{j}=-1}^{1} q_{j}\left(\zeta_{j}, \phi_{j}\right) \mathrm{d} \zeta_{j} \mathrm{~d} \phi_{j}=1
$$

where $\xi=\cos \theta$ and $\zeta_{j}=\cos \theta_{j}$.

The properties of the orientation distribution functions, $w(\xi, \phi, \eta)$ and $q_{j}\left(\zeta_{j}, \phi_{j}\right)$ can be examined in terms of a well-known mathematical procedure expanding the functions into infinite series of spherical harmonics, as fully discussed by Roe and Krigbaum $^{38-40}$ and by Nomura and Kawai. ${ }^{41}$ Thus,

$$
\begin{aligned}
w(\xi, \phi, \eta)= & \sum_{l=0}^{\infty} \sum_{m=-l}^{l} \sum_{n=-l}^{l} W_{l m n} Z_{l m n}(\xi) \\
& \times \exp [-i(m \phi+n \eta)] \\
q_{j}\left(\zeta_{j}, \phi_{j}\right)= & \sum_{l=0}^{\infty} \sum_{m=-l}^{l} Q_{l m}^{j} \Pi_{l}^{m}\left(\zeta_{j}\right) \exp \left(-i m \phi_{j}\right)
\end{aligned}
$$

where $Z_{l m n}(x)$ and $\Pi_{l}{ }^{m}(x)$ are normalized Jacobi's and Legendre's polynomials, respectively, interrelated by $\Pi_{l}^{m}(x)=Z_{l m 0}(x), l, m$, and $n$ are integers indicating the orders of the expansion, and $W_{l m n}$ and $Q_{l m}{ }^{j}$ are coefficients of each expanded series and are complex quantities given by

$$
\begin{gathered}
W_{l m n}=A_{l m n}+i B_{l m n} \\
Q_{l m}^{j}=\alpha_{l m}^{j}+i \beta_{l m}^{j}
\end{gathered}
$$

In turn, the coefficients of the expanded series can be represented by

$$
\begin{aligned}
& \left.\begin{array}{l}
A_{l m n} \\
B_{l m n}
\end{array}\right\}=\frac{1}{4 \pi^{2}} \int_{0}^{2 \pi} \int_{0}^{2 \pi} \int_{-1}^{1} w(\xi, \phi, \eta) Z_{l m n}(\xi) \\
& \times\left\{\begin{array}{l}
\cos (m \phi+n \eta) \\
\sin (m \phi+n \eta)
\end{array}\right\} \mathrm{d} \xi \mathrm{d} \phi \mathrm{d} \eta \\
& \left.\begin{array}{l}
\alpha_{l m}^{j} \\
\beta_{l m}^{j}
\end{array}\right\}=\frac{1}{2 \pi} \int_{0}^{2 \pi} \int_{-1}^{1} q_{j}\left(\zeta_{j}, \phi_{j}\right) \Pi_{l}^{m}\left(\zeta_{j}\right) \\
& \times\left\{\begin{array}{c}
\cos \left(m \phi_{j}\right) \\
\sin \left(m \phi_{j}\right)
\end{array}\right\} \mathrm{d} \zeta_{j} \mathrm{~d} \phi_{j}
\end{aligned}
$$

It is evident from eq 8 and 9 that the coefficients, $\left(A_{l m n}\right.$ and $\left.B_{l m n}\right)$ and $\left(\alpha_{l m}\right.$ and $\left.\beta_{l m}\right)$, are the averages of the distribution functions with respect to the spherical harmonics of the $l m n$-th and $l m$-th orders, respectively. In other words, the coefficients are explicitly the $l m n$-th and $l m$-th moments of the orientation distribution functions, and are very significant for defining the orientation factors, in 


\section{K. Fujita et al.}

general, as proposed by Nomura et al. ${ }^{41}$

Applying a generalization of the Legendre addition theorem to eq 1 , we obtain

$$
\begin{aligned}
\Pi_{l}^{m}\left(\zeta_{j}\right) \exp \left(i m \phi_{j}\right)= & \left(\frac{2}{2 l+1}\right)^{1 / 2} \sum_{n=-l}^{l} Z_{l m n}(\xi) \\
& \times \exp [i(m \phi+n \eta)] \\
& \times \Pi_{l}{ }^{n}\left(\cos \Theta_{j}\right) \exp \left(i n \Phi_{j}\right)
\end{aligned}
$$

Multiplying both sides of eq 10 by $w(\xi, \phi, \eta) \cdot q_{j}\left(\zeta_{j}, \phi_{j}\right)$, integrating over the ranges of the five angles, and taking eq 8 and 9 into account, we obtain

$$
\begin{aligned}
& \left.\begin{array}{l}
\alpha_{l m}^{j} \\
\beta_{l m}^{j}
\end{array}\right\}=2 \pi\left(\frac{2}{2 l+1}\right)^{1 / 2} \sum_{n=-l}^{l} \\
& \times\left\{\begin{array}{l}
A_{l m n} \cos \left(n \Phi_{j}\right)-B_{l m n} \sin \left(n \Phi_{j}\right) \\
A_{l m n} \sin \left(n \Phi_{j}\right)+B_{l m n} \cos \left(n \Phi_{j}\right)
\end{array}\right\} \Pi_{l}{ }^{n}\left(\cos \Theta_{j}\right)
\end{aligned}
$$

Equation 11 is one of the most significant results from the above mathematical analyses, interrelating not only the two coefficients, $Q_{l m}^{j}$ and $W_{l m n}$, each other, but also the coefficents $Q_{l m}^{j}$ of different $j$ with each other, and making it possible to determine the orientation distribution function $w(\xi, \phi, \eta)$ from a measurable function $q_{j}\left(\zeta_{j}, \phi_{j}\right)$ and further from $Q_{m}{ }^{j}$ to an approximation depending upon the choice of j's.

The functions, $Z_{l m n}(x)$ and $\Pi_{l}^{m}(x)$, are symmetric with respect to $m, n$ and $x$. The orientation distribution function, $q_{j}\left(\zeta_{j}, \phi_{j}\right)$ also has symmetry, such as orthogonal-biaxial symmetry with respect to the Cartesian coordinate system $0-x_{1} x_{2} x_{3}$ or uniaxial symmetry with respect to the $x_{3}$ axis for most industrial products of sheet-like or fibrous materials, respectively, providing that the Cartesian coordinate $0-x_{1} x_{2} x_{3}$ is properly fixed within the specimen, as suggested above. In addition, the structural unit (crystallite) has symmetry, though not always, such as orthorhombic or cylindrical symmetry with respect to the Cartesian coordinate system $0-u_{1} u_{2} u_{3}$, again providing that the coordinate system $0-u_{1} u_{2} u_{3}$ is properly fixed within the structural unit. These symmetries make the general description given by eq 5, 6, and 11 much simpler, mainly because of the vanishing coefficients of $A_{l m n}$ and $\alpha_{l m}^{j}$ with odd $l, m$, or $n$ and the coefficents of all of $B_{l m n}$ and $\beta_{l m}^{j}$ from the equations, and partly because of the symmetric nature of the remaining coefficients with respect to the sign of $m$ and $n$.

Below are shown some simplified results obtained under combinations of particular symmetries of the distribution function $q_{j}$ and the structural unit. The orthogonal-biaxial symmetry of $q_{j}$ gives the coefficients values other than zero when $l$ and $m$ are even. The uniaxial symmetry of $q_{j}$ also gives the coefficients values rather than zero when $l$ is even and $m$ is zero. The orthorhombic symmetry of the structural unit makes the coefficients take on values other than zero when $n$ is even. The cylindrical symmetry of the unit causes the coefficients values other than zero when $n$ is zero. Thus, in the following, eq 5, 6, and 11 have been simplified for the uniaxial symmetry of $q_{j}$ with the orthorhombic crystallites with which we are concerned in the present study.

$$
\begin{aligned}
& w(\xi, 0, \eta)=\sum_{l=0}^{\infty} A_{l 00} \Pi_{l}(\xi) \\
& +2 \sum_{l=2}^{\infty} \sum_{n=2}^{l} A_{l 0 n} \Pi_{l}^{n}(\xi) \cos (n \eta) \\
& q_{j}\left(\zeta_{j}, 0\right)=\sum_{l=0}^{\infty} \alpha_{l 0}^{j} \Pi_{l}\left(\zeta_{j}\right) \\
& A_{l 0 n}=\frac{1}{2 \pi} \int_{0}^{2 \pi} \int_{-1}^{1} w(\xi, 0, \eta) \Pi_{l}^{n}(\xi) \cos (n \eta) \mathrm{d} \xi \mathrm{d} \eta \\
& \alpha_{l 0}^{j}=\int_{-1}^{1} q_{j}\left(\zeta_{j}, 0\right) \Pi_{l}\left(\zeta_{j}\right) \mathrm{d} \zeta_{j} \\
& \alpha_{l 0}^{j}=2 \pi\left(\frac{2}{2 l+1}\right)^{1 / 2}\left[A_{l 00} \Pi_{l}\left(\cos \Theta_{j}\right)\right. \\
& \left.+2 \sum_{n=2}^{l} A_{l o n} \Pi_{l}^{n}\left(\cos \Theta_{j}\right) \cos \left(n \Phi_{j}\right)\right]
\end{aligned}
$$

The uniaxial orientation distribution function of the $j$-th crystal plane, $q_{j}\left(\zeta_{j}, 0\right)$, can be obtained from the X-ray diffraction intensity distribution at a given twice the Bragg angle of the plane, $I_{j}\left(\theta_{j}, 0\right)$, as follows:

$$
q_{j}\left(\zeta_{j}, 0\right)=\frac{I_{j}\left(\theta_{j}, 0\right)}{4 \pi \int_{0}^{\pi / 2} I_{j}\left(\theta_{j}, 0\right) \sin \theta_{j} \mathrm{~d} \theta_{j}}
$$

Once the $q_{j}\left(\zeta_{j}, 0\right)$ is determined, $\alpha_{l 0}^{j}$ can be calculated from eq 15 for any even $l$. On the other hand, the polar and azimuthal angles, $\Theta_{j}$ and $\Phi_{j}$, of the reciprocal lattice vector of the $j$-th crystal plane with 
respect to the Cartesian coordinate system $0-u_{1} u_{2} u_{3}$ fixed properly within polyethylene crystal (orthorhombic crystal), can be calculated by

$$
\begin{aligned}
& \cos \Theta_{j}=\frac{(l / c)}{\left[(h / a)^{2}+(k / b)^{2}+(l / c)^{2}\right]^{1 / 2}} \\
& \cos \Phi_{j}=\frac{(h / a)}{\left[(h / a)^{2}+(k / b)^{2}\right]^{1 / 2}}
\end{aligned}
$$

where $h, k$, and $l$ are the Miller indices of the $j$-th crystal plane, and $a, b$, and $c$ are lattice constants of polyethylene orthorhombic crystal along the crystal $a-, b$-, and $c$-axis, respectively, which are equal to 7.41, 4.94, and $2.55 \AA$.

With $\alpha_{l 0}^{j}$ and $\Theta_{j}$ and $\Phi_{j}$ thus determined for the $j$ th crystal plane, the coefficient $A_{l 0 n}$ can be calculated by solving simultaneous equations of eq 16 changing $j$ up to at least $((l / 2)+1)$. In turn, the orientation distribution function of the crystallites, $w(\xi, 0, \eta)$ can be determined from eq 12 with the approximation of finite series of expansion up to $n=2(j-1)$, instead of the infinite series of expansion. From experimental view point, however, the measuring accuracy of $I_{j}\left(\theta_{j}, 0\right)$ and, consequently, that of $q_{j}\left(\zeta_{j}, 0\right)$ varies with $j$, so that the simultaneous equations must be solved by use of a weightened least-squares method, as proposed by Roe and Krigbaum. ${ }^{38-40}$

\section{TEST SPECIMEN AND EXPERIMENTAL PROCEDURE}

Commercial pellets of a high-density polyethylene (Sholex 6009, Japan Olefin Chemical Ind., Ltd.) having a viscosity-average molecular weight of 67,000 and a degree of branching of $0.7 \mathrm{CH}_{3} / 1000$ carbons were placed in a spacer sandwiched between two polished stainless steel plates, and molten at $180^{\circ} \mathrm{C}$ in a laboratory hot press for $10 \mathrm{~min}$. A pressure of $200 \mathrm{~kg} \mathrm{~cm}^{-2}$ was applied for another $10 \mathrm{~min}$ at the same temperature, and the melt was slowly cooled down to $125 \mathrm{C}$, and then kept for $2 \mathrm{~h}$ at $125^{\circ} \mathrm{C}$. The stainless steel plates containing the melt were removed from the press and immediately plunged into ice water to obtain a quenched specimen in sheet form. This quenched specimen was annealed at $105^{\circ} \mathrm{C}$ for $4 \mathrm{~h}$ in a vacuum oven and gradually cooled down to ambient temperature. The thickness of the sheet specimen was adjusted by the spacer to $500 \mu \mathrm{m}$ for X-ray diffraction measurements. By polarized light-scattering investigations, the annealed specimen was found to have a welldeveloped spherulitic crystalline texture whose $H_{\mathrm{v}}$ and $V_{\mathrm{v}}$ patterns are shown in Figure 2. The average size of the spherulites was estimated from the $H_{\mathrm{v}}$ pattern to be about $14 \mu \mathrm{m}$ in radius. The density of

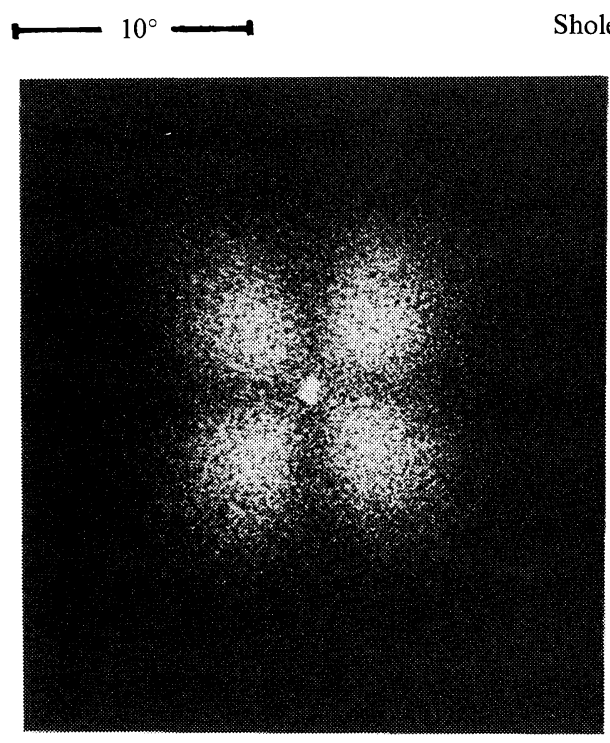

$H_{\mathrm{v}}$
Sholex 6009 (A)

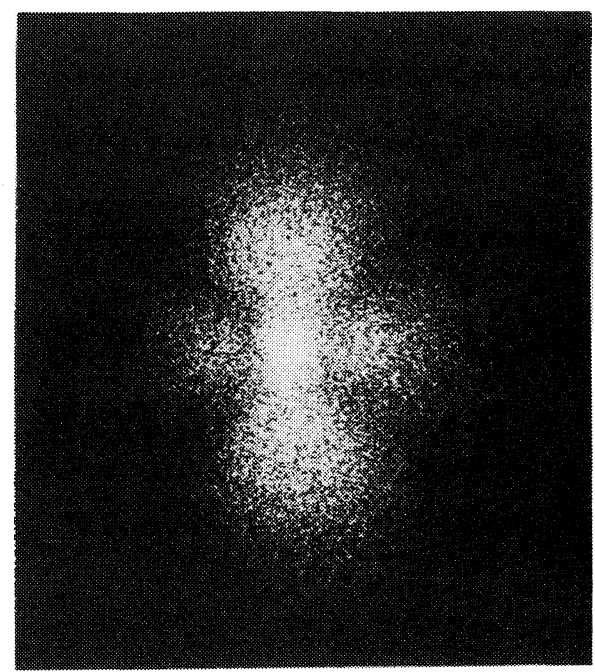

$V_{\mathrm{v}}$

Figure 2. Small-angle light scattering patterns from an isotropic film specimen of a high-density polyethylene used under $H_{\mathrm{v}}$ and $V_{\mathrm{v}}$ polarization conditions. 


\section{K. FuJiTA et al.}

the annealed specimen was determined to be $0.957 \mathrm{~g} \mathrm{~cm}^{-3}$ by a methanol-water density gradient column at $25.0^{\circ} \mathrm{C}$, and the degree of crystallinity was estimated from the density value to be $71 \%$ in volume fraction and $74 \%$ in weight fraction, with densities of the crystalline and noncrystalline regions being 1.000 and $0.852 \mathrm{~g} \mathrm{~cm}^{-3}$, respectively.

The annealed sheet specimen was cut into a ribbon of $10 \mathrm{~cm}$ long and $1.5 \mathrm{~cm}$ wide. The ribbon was kept at an initial gauge length of $8 \mathrm{~cm}$ and stretched uniaxially in $60^{\circ} \mathrm{C}$ warm water to extension ratios of $1.1,1.2,1.3$, and 1.4 at extension rate as low as $1.5 \% / \mathrm{min}$. The extension ratio of $1.4 \mathrm{was}$ the limit of uniform deformation beyond which the specimen exhibited macroscopic necking. The stretched specimens thus obtained were clamped between two metalic flanges and kept at an ambient temperature for about a week until the X-ray diffraction measurements were carried out with the $\mathrm{Cu}-\mathrm{K} \alpha$ line of the $\mathrm{X}$-ray beam generated from a rotating anode type generator (RU-200Z, Rigaku Denki Co., Tokyo) with a power of $40 \mathrm{kV}$ and $200 \mathrm{~mA}$. The measurements of X-ray diffraction intensity distribution were performed for 13 different crystal planes, as listed in Table I, using an ordinary horizontal scanning type goniometer operating at a fixed time step scan of 0.1 or $1^{\circ}$ interval over a range of twice the Bragg angle $2 \theta_{\mathbf{B}}$ from 12 to

Table I. Bragg angle $2 \theta_{\mathrm{B}}$ and polar and azimuthal angles $\Theta_{j}$ and $\Phi_{j}$ in regard to the orientation of reciprocal lattice vector of the $j$-th crystal plane within polyethylene crystal

\begin{tabular}{cccc}
\hline$(h k l)$ & $2 \theta_{\mathbf{B}}$ & $\Theta_{j}$ & $\Phi_{j}$ \\
\hline$(110)$ & 21.62 & 90.00 & 56.31 \\
$(200)$ & 24.02 & 90.00 & 0.00 \\
$(210)$ & 30.15 & 90.00 & 36.87 \\
$(020)$ & 36.38 & 90.00 & 90.00 \\
$(120)$ & 38.42 & 90.00 & 71.57 \\
$(011)$ & 39.79 & 27.30 & 90.00 \\
$(310)$ & 40.85 & 90.00 & 26.57 \\
$(111)$ & 41.69 & 31.81 & 56.31 \\
$(201)$ & 43.07 & 34.54 & 0.00 \\
$(220)$ & 44.06 & 90.00 & 56.31 \\
$(211)$ & 47.01 & 40.71 & 36.87 \\
$(311)$ & 55.00 & 49.10 & 26.57 \\
$(002)$ & 74.40 & 0.00 & - \\
\hline
\end{tabular}

$76^{\circ}$, with the $x_{1}$ axis of the specimen tilted by an angle of $\theta_{\mathbf{B}}$ with respect to the incident $X$-ray beam. The horizontal scanning was carried out at fixed angles by rotating stepwise the specimen about the $x_{1}$ axis at $5^{\circ}$ intervals so that the orientation distribution function of reciprocal lattice vectors of the $j$-th crystal planes at a particular azimuthal angle of $90^{\circ}$ might be obtained. The function $q_{j}\left(\zeta_{j}, \phi_{j}=90^{\circ}\right)$, thus obtained, is sufficient for cylindrically symmetric specimen of uniaxially stretched in the $x_{3}$ direction.

In practice, the intensity distribution was first measured as a function of $2 \theta_{\mathbf{B}}$ at a given rotational angle $\psi_{0}$ of the specimen about the $x_{1}$ axis; corrections were made for air-scattering, background. noise, polarization, absorption, and incoherent scattering. The corrected intensity distribution, which includes not only crystalline contributions from respective $j$-th crystal planes, but also noncrystalline contributions, may be written as ${ }^{29}$

$$
\begin{aligned}
I\left(2 \theta_{\mathrm{B}}, \psi_{0}\right) & =I_{\text {crys }}\left(2 \theta_{\mathrm{B}}, \psi_{0}\right)+I_{a m}\left(2 \theta_{\mathrm{B}}, \psi_{0}\right) \\
& =\sum_{i}^{j, a m} I_{\max , i}\left(\psi_{0}\right) \exp \left[-\frac{\left(2 \theta_{\mathrm{B}}-2 \theta_{\mathrm{B}, i}\right)^{2}}{2 \sigma_{i}^{2}\left(\psi_{0}\right)}\right](20)
\end{aligned}
$$

Here, the intensity distribution is assumed to be the sum of the Gaussian type functions appearing at $2 \theta_{\mathrm{B}, i}$ with maximum intensity $I_{\max , i}\left(\psi_{0}\right)$ and broadening factor $\sigma_{i}\left(\psi_{0}\right)$ for each of crystalline and noncrystalline contributions. The separation of these contributions and, subsequently, the determinations of $I_{\max , i}\left(\psi_{0}\right), \quad \sigma_{i}\left(\psi_{0}\right)$ and $2 \theta_{\mathrm{B}, i}$ from $I\left(2 \theta_{\mathrm{B}}, \psi_{0}\right)$ were performed by a trial and error method as a function of the rotating angle $\psi$, with the conclusion that $\sigma_{j}(\psi)$ was almost constant. $I_{\text {max }, j}(\psi)$ thus determined is proportional to $I_{j}\left(\theta_{j}, 0\right)$ in eq 17 , and the orientation distribution function $q_{j}\left(\zeta_{j}, 0\right)$ can be determined from the equation for the respective $j$-th crystal planes.

\section{EXPERIMENTAL RESULTS AND DISCUSSION}

\section{Uniaxial Orientation Behavior of Polyethylene Crystals}

Figure 3 shows the uniaxial orientation distribution functions $q_{j}\left(\zeta_{j}, 0\right)$, thus determined, for a specimen stretched to an extension ratio of 1.4. 12 different $j$-th crystal planes were observed except for the (220) crystal plane which is the 2 nd order 


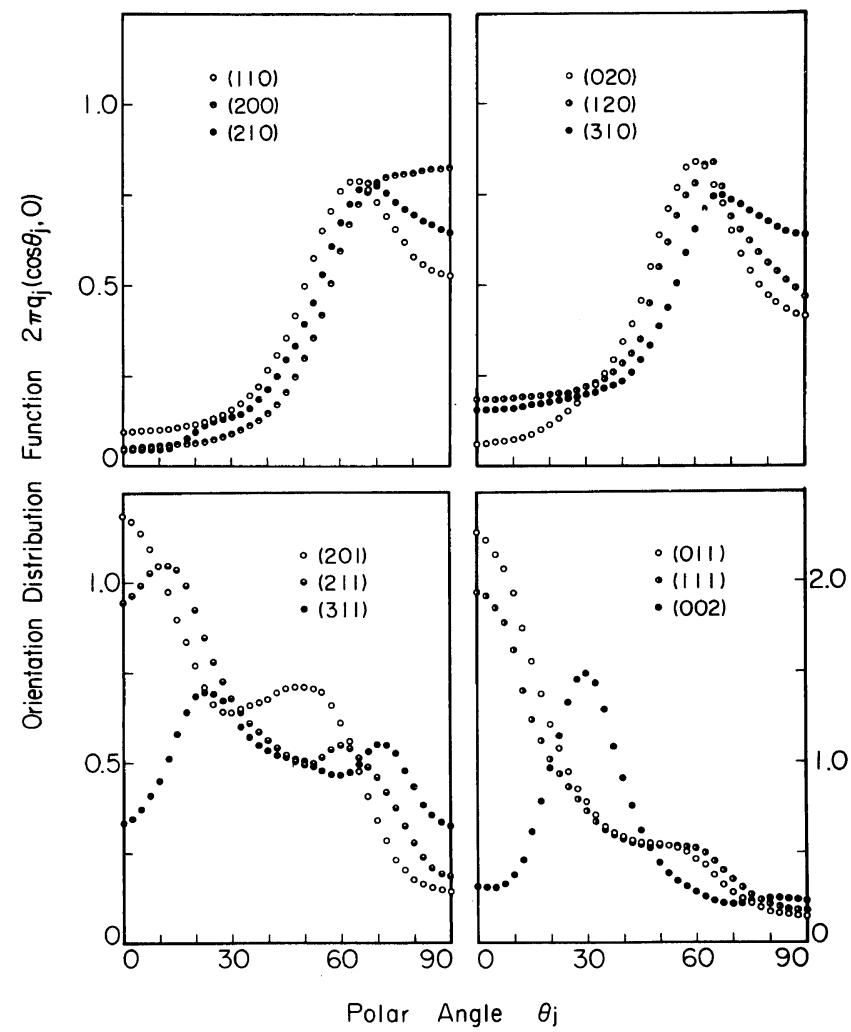

Figure 3. Uniaxial orientation distribution functions $q_{j}\left(\zeta_{j}, 0\right)$ for the $j$-th crystal planes determined from $\mathrm{X}$-ray diffraction intensity distributions at a given extension ratio of 1.4 .

reflection of the (110) crystal plane. With $q_{j}\left(\zeta_{j}, 0\right)$ for $j=12$, the coefficients of $\alpha_{10}^{j}$ were calculated from eq 15 for any higher-orders of $l$, and then the coefficients $A_{l 0 n}$ were calculated from the simultaneous equations of eq 16 with respect to $j$ by the weightened least-squares method with a weightening factor $\rho_{j}$, where $l$ was limited up to 18 , instead of $22[=2(j-1)]$, in this particular case. ${ }^{* 1}$ The weightening factor $\rho_{j}$ may be defined as the reciprocal of the measuring error of the $j$-th crystalline diffraction $P_{j}$ and is represented by

$$
p_{j}=\frac{\delta_{j}}{\gamma_{j}}=\frac{\left[\left(\gamma_{T} / t_{T}\right)+\left(\gamma_{a} / t_{a}\right)\right]^{1 / 2}}{\gamma_{j}}=\frac{\left[\gamma_{T}+\left(\gamma_{T} \gamma_{a}\right)^{1 / 2}\right]^{1 / 2}}{\gamma_{j}\left(t_{T}\right)^{1 / 2}}
$$

where $\delta_{j}$ is the standard derivation of the counting rates for the $j$-th crystalline diffraction peak intensity, $\gamma$ and $t$ are the counting rate and counting
Table II. Values of weight-factor $\rho_{j}$ for the $j$-th crystal plane at various extension ratios $\lambda$

\begin{tabular}{lllll}
\hline & \multicolumn{5}{c}{$\rho_{j}$} \\
\cline { 2 - 5 }$(h k l)$ & $\lambda=1.1$ & $\lambda=1.2$ & $\lambda=1.3$ & $\lambda=1.4$ \\
\hline & & & \\
$(110)$ & 1.000 & 1.000 & 1.000 & 1.000 \\
$(200)$ & 0.596 & 0.576 & 0.560 & 0.559 \\
$(210)$ & 0.122 & 0.138 & 0.138 & 0.208 \\
$(020)$ & 0.185 & 0.199 & 0.201 & 0.207 \\
$(120)$ & 0.031 & 0.033 & 0.032 & 0.035 \\
$(011)$ & 0.248 & 0.331 & 0.373 & 0.433 \\
$(310)$ & 0.163 & 0.170 & 0.165 & 0.162 \\
$(111)$ & 0.183 & 0.243 & 0.269 & 0.303 \\
$(201)$ & 0.216 & 0.251 & 0.246 & 0.253 \\
$(220)$ & 0.087 & 0.096 & 0.094 & 0.095 \\
$(211)$ & 0.138 & 0.154 & 0.149 & 0.161 \\
$(311)$ & 0.109 & 0.112 & 0.103 & 0.106 \\
$(002)$ & 0.125 & 0.166 & 0.180 & 0.193 \\
\hline
\end{tabular}

*1 The limitation of $l$ up to 18 , instead of 22 , was adopted mainly because a better convergence of the least-squares method could be obtained by exclusion of certain crystal planes for which measurements of $q_{j}\left(\zeta_{j}, 0\right)$ were less accurate, and partly because non-serious effect of the termination error upon calculating $A_{l 0 n}$ could be resulted. 
K. Fujita et al.
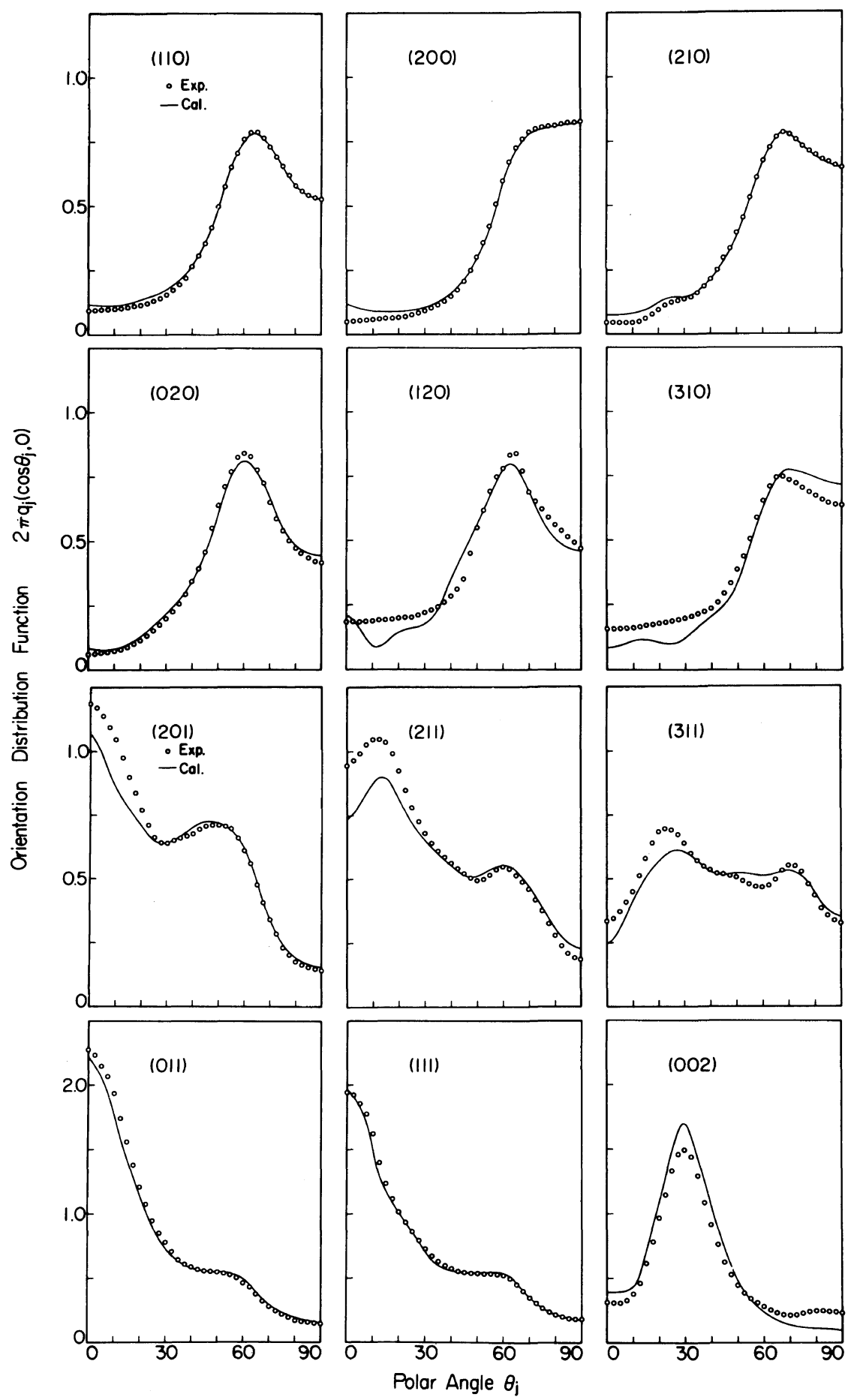

Figure 4. Comparison of observed uniaxial orientation distribution functions $q_{j}\left(\zeta_{j}, 0\right)$ (open circles) for the $j$-th crystal planes with those calculated (solid lines) at a given extension ratio of 1.4 . 
time, respectively, and the subscripts, $T, j$, and $a$ denote the total, $j$-th crystalline and background components, respectively, all at $2 \theta_{\mathrm{B}, j}$. The weightening factors thus determined for the respective crystal planes are listed in Table II. These are values relative to that of the (110) crystal plane at various extension ratios.

Figure 4 compares the observed orientation distribution functions with that calculated for respective crystal planes at the extension ratio of 1.4. That is, we recalculated $\alpha_{l 0}^{j}$ in turn from $A_{l 0 n}$, which were initially determined by the weightened leastsquares method, by the use of eq 16 , and further calculated $q_{j}\left(\zeta_{j}, 0\right)$ from the recalculated $\alpha_{l 0}^{j}$ by the use of eq 13. As can be seen in the figure, fairly good agreement between the observed and calculated distribution functions was obtained even for the less accuratly measured crystal planes with lower weightening factor, such as the (311), (120), and (002) crystal planes. This suggests a high reliability of the $A_{l 0 n}$ thus determined. A meansquare error between the calculated $\alpha_{l 0}^{j}$ and recalculated $\alpha_{10}^{j}$ may be defined as

$$
R=\frac{\sum_{j, l} \rho_{j}\left[\left(\alpha_{l 0}^{j}\right)_{\mathrm{cal}}-\left(\alpha_{l 0}^{j}\right)_{\mathrm{recal}}\right]^{2}}{\sum_{j, l} \rho_{j}\left[\left(\alpha_{l 0}^{j}\right)_{\mathrm{cal}}\right]^{2}}
$$

Table III. Mean-square error $R$ at various extension ratios $\lambda$

\begin{tabular}{lllll}
\hline$\lambda$ & 1.1 & 1.2 & 1.3 & 1.4 \\
\hline$R$ & 0.036 & 0.022 & 0.030 & 0.020 \\
\hline
\end{tabular}

$4 \pi^{2} w(\xi, 0, \lambda)$
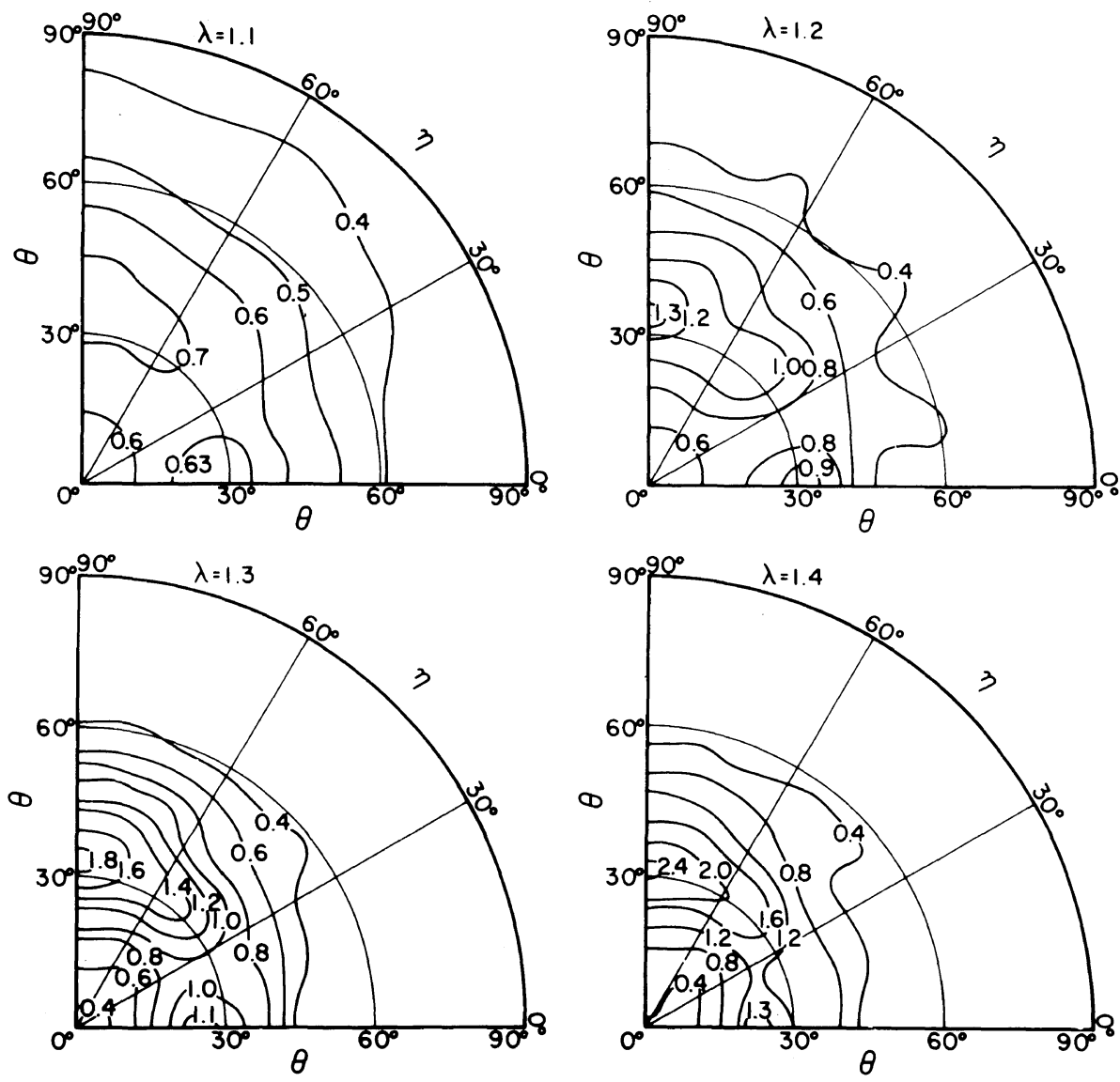

Figure 5. Change in uniaxial orientation distribution function of crystallites (crystal grains), $w(\xi, 0, \eta)$ with an extension ratio of the bulk specimen $\lambda$. 
As listed in Table III, the mean-square error is no more than a few percent at each extension ratio, indicating the high reliability of $A_{l 0 n}$ again. These values of $R$ are much smaller than the 10 and 30 percent obtained in the studies of uniaxially stretched polyethylenes by Krigbaum and $\mathrm{Roe}^{39}$ and by Matsuo et al., ${ }^{23}$ respectively.

Figure 5 shows the change in the uniaxial orientation distribution function of crystallites, $w(\xi, 0, \eta)$ with the extension ratio. This function was calculated using eq 12 with a finite series of expansion with the coefficients $A_{l 0 n}$ determined from eq 16 with $l$ limited up to 18 . As can be seen from the figure, two populous regions appear in the orientation distribution; i.e., one at $\theta$ a little larger than $30^{\circ}$ and $\eta$ of $90^{\circ}$, and the other at $\theta$ a little smaller than $30^{\circ}$ and $\eta$ of $0^{\circ}$. It seems that the former population appears posteriorly but at much greater rate than the latter population, suggesting different mechanisms of spherulite defermation to yield the two populations.

Actually, as was pointed out in previous studies on the deformation mechanism of polyethylene spherulites, ${ }^{7,18,19}$ the appearance of two populations in $w(\xi, 0, \eta)$ must be explained in terms of two types of preferential reorientation of crystallites (crystal grains) within orienting lamellae being accompanied with the uniaxial deformation of spherulites; i.e., rotations of the crystal grains about their own crystal $a$ - and $b$-axes, respectively, in association with lamellar shearing and detwisting mostly at the polar and equatorial zones of the spherulites, both so as to align their crystal $c$-axes (molecular axes) toward the stretching direction $\left(x_{3}\right.$ axis) as a result of the straining of tie-chain molecules between adjacent lamellae. The above concept of rotations of the crystal grains about the crystal $a$ - and $b$-axis does not necessarily invoke a specific nature for the plasticity of polyethylene crystal, but a hypothetical concept of paracrystalline nature of the crystal lamella. That is, the lamella must consist of "aggregates of microparacrystallite domains" (crystal grains) ${ }^{27}$ or of the so-called crystal mosaic blocks, capable of rotating at the grain boundaries.

The above explanation may be demonstrated schematically in terms of the orientation distribution function $w(\xi, 0, \eta)$ for some particular cases of reorientation of the crystal grains within crystal lamellae. First, consider crystal lamellae orienting in cylindrical symmetry about the $x_{3}$ axis with lamellar axes inclined from the $x_{3}$ axis by a given angle of 0 , $30,45,60$ or $90^{\circ}$, and the crystal grains orienting within each lamella with their $b$-axes parallel to the lamellar axis but with their $a$ - and $c$-axes rotating periodically about the lamellar axis in association with periodic twisting of the lamella. The orientation distributions of the crystal grains may thus be represented, respectively, by the point 1 at $\theta=90^{\circ}$ and $\eta=90^{\circ}$, the curves 2,3 , and 4 , and the straight line 5 in Figure 6(a). If the crystal grains within each lamella rotate about their own $b$-axes (lamellar axis) to allow their $c$-axes to orient toward the plane including the $x_{3}$ axis and the lamellar axis, the orientation distribution must be displaced, as indicated by the arrows in Figure 6(b), and must be concentrated toward the points at $\theta=0 \sim 90^{\circ}$ and $\eta=90^{\circ}$. On the other hand, if the crystal grains within crystal lamella inclined from the $x_{3}$ axis by a given angle $\theta_{0}$, for example $45^{\circ}$, rotate about their own $a$-axes so as to orient their $c$-axes toward the plane including the $x_{3}$ axis and the lamellar axis or just toward the $x_{3}$ axis, if the $c$-axis is already in the plane, then the orientation distribution must be displaced, as indicated by the arrows in Figure 6(c), and must be concentrated toward the points at $\theta=$ $0 \sim \theta_{0}\left(=45^{\circ}\right)$ and $\eta=0^{\circ}$.

The crystal rotation about the crystal $a$ - or $b$-axis can be accentuated for the lamellae in the polar or equatorial zone of spherulites, and the orientation distribution of the crystal grains must shift, as indicated by the arrows in Figure 6(d) or Figure 6(e), so as to be concentrated in some regions around $\eta \simeq 90$ or $0^{\circ}$ and small values of $\theta$ depending on the degree of crystal rotation. For the crystal grains within the crystal lamellae inclined from the $x_{3}$ axis by angles intermediate between the polar and equatorial zones, the two types of crystal rotations must interact and become superposed on each other, probably at first about the crystal $b$-axis and then about the crystal $a$-axis, as indicated by the arrows in Figure 6(f), so as to orient the crystal $c$ axes parallel to the stretching direction of $x_{3}$. If the change in the orientation distribution of the crystal lamellae and the crystal rotation mechanisms, as given by Figure 6(d) through Figure 6(f), are considered, the population in the orientation distribution of the crystal grains at $\theta \simeq 30^{\circ}$ and $\eta=90^{\circ}$ can be understood as resulting from the crystal rotation mechanism about the crystal $a$-axis, as 


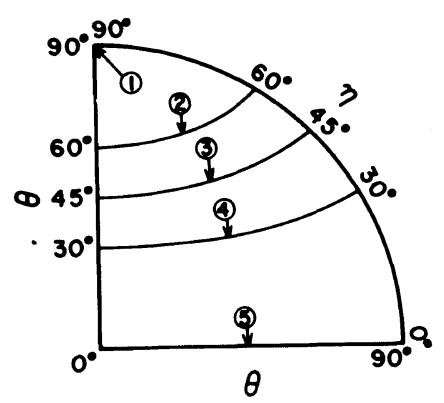

(a)

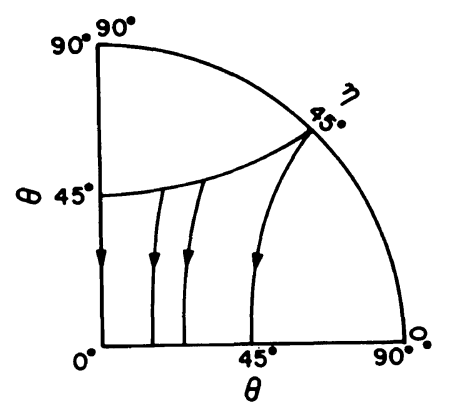

(c)

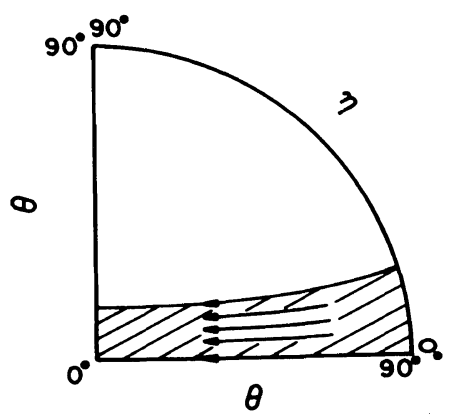

(e)

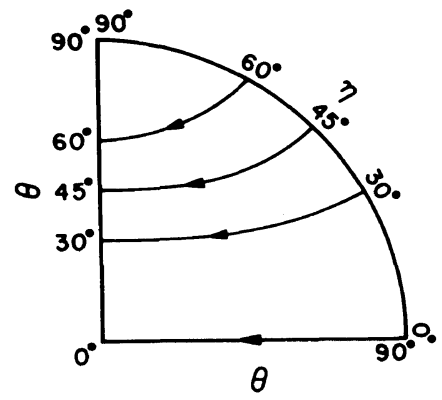

(b)

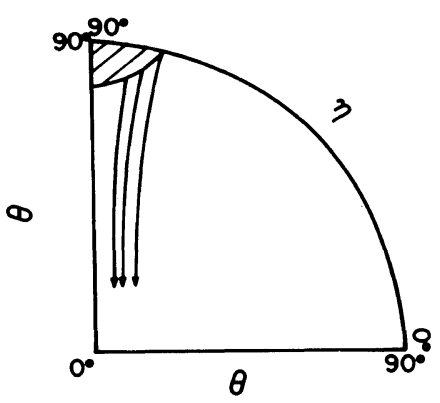

(d)

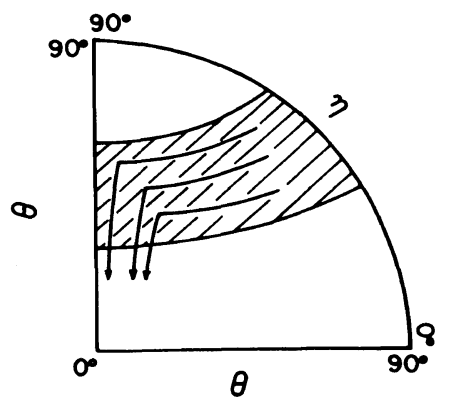

(f)

Figure 6. Schematic diagrams demonstrating the uniaxial orientation distribution function of crystal grains, $w(\xi, 0, \eta)$ for a particular lamellar orientation and its change with reorientation of the crystal grains within the lamellae: (a) $w(\xi, 0, \eta)$ s for a particular lamellar orientations with polar angles of $\theta^{\prime}=0^{\circ}$ (point 1), $\theta^{\prime}=30-60^{\circ}$ (curve $2-4$ ), and $\theta^{\prime}=90^{\circ}$ (straight line 5): (b) changes of $w(\xi, 0, \eta)$ s in (a) with rotation of the grains about their own $b$-axes (lamellar axis): (c) change in $w(\xi, 0, \eta)$ for a particular lamellar orientation, for example, with polar angle of $\theta^{\prime}=45^{\circ}$ with rotation of the grains about their own $a$-axes: (d) change in $w(\xi, 0, \eta)$ with rotation of the grains around $a$-axes for lamellae at polar zone of spherulite: (e) change in $w(\xi, 0, \eta)$ with rotation of the grains around $b$-axes for lamellae at equatorial zone of spherulite: and (f) changes in $w(\xi, 0, \eta)$ s for lamellae orienting with intermediate angles of $\theta^{\prime}$ around $45^{\circ}$ in association with rotations of the grains at first around $b$-axes and then around $a$-axes. 
given by Figures 6(d) and 6(f). This population may be much more prominent than the population at $\theta \simeq 30^{\circ}$ and $\eta=0^{\circ}$ which results from the crystal rotation mechanism about the crystal $b$-axis, as given by Figure 6(e). The limitation of $\theta$, the $c$-axis orientation angle to the stretching direction, down to about $30^{\circ}$ does not seem to be significant, but merely a result of the condition that this particular specimen was stretched to an extension ratio of 1.4, beyond which the macroscopic necking accompanied by disintegration of spherulitic crystalline texture takes place. Actually, if the spherulitic crystalline texture is transformed into the so-called fiber structure, accompanied by highly uniaxial deformation and disintegration of the spherulitic texture, the orientation distribution of the crystal grains must be concentrated in the region of $\theta$ to become nearly as small as zero.

\section{Calculation of Orientation Distribution Function of}

Polyethylene Crystals from Spherulite Deformation Model

The orientation distribution function of crystal grains can be calculated using a spherulite deformation model. By a comparison of the calculated result with that observed, the deformation mechanism can be evaluated in terms of the model parameters. Figure 7 shows the orientation of a

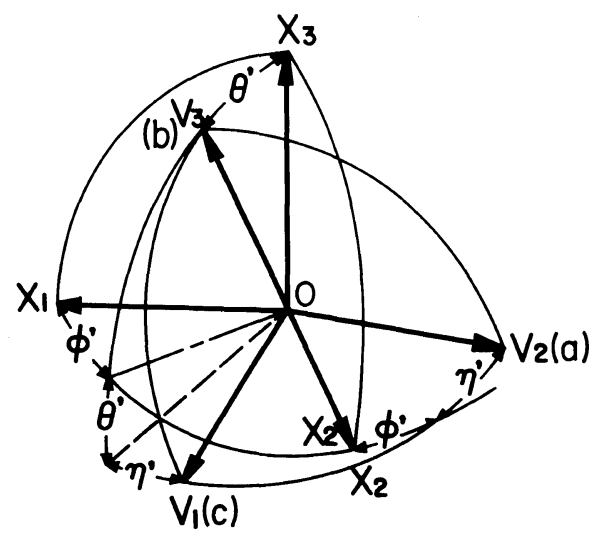

Figure 7. Three Euler angles, $\phi^{\prime}, \theta^{\prime}$, and $\eta^{\prime}$, specifying the orientation of a Cartesian coordinate system of 0 $v_{1} v_{2} v_{3}$ fixed within a segment of crystal lamella with respect to the Cartesian coordinate system $0-x_{1} x_{2} x_{3}$ fixed within the bulk specimen. The coordinate $0-v_{1} v_{2} v_{3}$ is fixed to the lamellar segment in such a way that the $v_{1}$ and $v_{3}$ are normal to the lamellar surface and parallel to the lamellar axis, respectively.
Cartesian coordinate system $0-v_{1} v_{2} v_{3}$ fixed within a crystal lamella relative to the Cartesian coordinate system $0-x_{1} x_{2} x_{3}$ fixed within the bulk specimen in terms of three Euler angles $\phi^{\prime}, \theta^{\prime}$, and $\eta^{\prime}$. The Cartesian coordinate $0-v_{1} v_{2} v_{3}$ may be fixed within a segment of crystal lamella in such a way that the $v_{1}$ and $v_{3}$ are normal to the lamellar surface and parallel to the lamellar axis, respectively. Assuming the uniaxial deformation of polyethylene spherulite to follow an affine deformation hypothesis keeping its volume constant, as has been postulated by several authors, ${ }^{2-5,12,15,18,19,23}$ the radius of the uniaxially deformed spherulite may be given as a function of orientation angle $\theta^{\prime}$ by the equation:

$$
g\left(\theta^{\prime}\right)=\frac{\lambda}{\left[\lambda^{3}-\left(\lambda^{3}-1\right) \cos ^{2} \theta^{\prime}\right]^{1 / 2}}
$$

where $\lambda$ is the extension ratio of the spherulite in the $x_{3}$ direction.

Assuming that each crystal lamella consists of same number of crystal grains and that the orientation of the crystal grains within each lamellar occurs so that their $b$-axes are parallel to the lamellar axis, irrespective of lamellar orientation, then the number of crystal lamellae and, consequently, the number of crystal grains orienting in a range from $\theta^{\prime}$ to $\left(\theta^{\prime}+\mathrm{d} \theta^{\prime}\right)$ may be given by

$$
N\left(\theta^{\prime}\right)=\frac{N_{0} \lambda^{3}}{2\left[\lambda^{3}-\left(\lambda^{3}-1\right) \cos ^{2} \theta^{\prime}\right]^{3 / 2}}
$$

where $N_{0}$ is the total number of crystal grains within a spherulite. Thus, the orientation distribution of the crystal grains can be normalized as

$$
N\left(\theta^{\prime}\right) / N_{0}=\left[g\left(\theta^{\prime}\right)\right]^{3} / 2
$$

Let us formulate the following four types of reorientation mechanisms of the crystal grains within the orienting lamellae.

(1) Type Ca: Rotation of the crystal grains about their own $a$-axes. The orientation distribution function of the crystal grains may be formulated as

$$
\begin{aligned}
P_{\mathrm{Ca}}(\theta, \eta) & =\frac{W_{\mathrm{Ca}}}{8 \pi^{2}} \\
& \times\left[\frac{\cos ^{2} \theta\left\{2 \cos ^{2}\left(\theta_{0, \mathrm{Ca}}\right)-\cos ^{2} \theta\right\}}{\cos ^{4}\left(\theta_{0, \mathrm{Ca}}\right)}\right]^{2\left(J_{\mathrm{a}}-1\right)} \\
& \times(\sin \eta)^{2\left(I_{\mathrm{a}}-1\right)}
\end{aligned}
$$

(2) Type $C b$ : Rotation of the crystal grains about their own $b$-axes. The orientation distribution 
function of the crystal grains may be formulated as.

$$
\begin{aligned}
P_{\mathrm{Cb}}(\theta, \eta)= & \frac{W_{\mathrm{Cb}}}{8 \pi^{2}} \\
& \times\left[\frac{\cos ^{2} \theta\left\{2 \cos ^{2}\left(\theta_{0, \mathrm{Cb}}\right)-\cos ^{2} \theta\right\}}{\cos ^{4}\left(\theta_{0, \mathrm{Cb}}\right)}\right]^{2\left(J_{\mathrm{b}}-1\right)} \\
& \times(\cos \eta)^{2\left(I_{\mathrm{b}}-1\right)}
\end{aligned}
$$

(3) Type B: Orientation of the crystal grains without any rotation within the lamella. The orientation distribution function of the grains may be given, similarly to eq 24 , as

$$
P_{\mathbf{B}}\left(\theta^{\prime}, \eta^{\prime}\right)=\frac{W_{\mathbf{B}}}{8 \pi^{2}} \frac{\lambda^{3}}{\left[\lambda^{3}-\left(\lambda^{3}-1\right) \cos ^{2} \theta^{\prime}\right]^{3 / 2}}
$$

(4) Type R: Random orientation of the crystal grains representing the degree of imperfection in the spherulitic crystalline texture. The orientation distribution function is given by

$$
P_{\mathrm{R}}(\theta, \eta)=1 / 8 \pi^{2}
$$

where $W_{i}(i=\mathrm{Ca}, \mathrm{Cb}$, and $\mathrm{B})$ are normalization constants, $\theta_{0, i}(i=\mathrm{Ca}$ and $\mathrm{Cb})$ are the angles at which the respective orientation distribution functions, $P_{\mathrm{Ca}}$ and $P_{\mathrm{Cb}}$, exhibit maximum intensities, $J_{i}$ $(i=\mathrm{a}$ and $\mathrm{b})$ are the parameters representing the sharpness of the orientation distribution of the angle $\theta$ at the vicinity of $\theta_{0, i}$ for the $P_{\mathrm{Ca}}$ and $P_{\mathrm{Cb}}$, respectively, and $I_{i}(i=\mathrm{a}$ and $\mathrm{b})$ are the parameters representing the sharpness of distribution of the rotational angle $\eta$ of crystal grains about their own $c$-axes for $P_{\mathrm{Ca}}$ and $P_{\mathrm{Cb}}$, respectively.

In the undeformed state, the spherulite must be composed of the crystal grains of Type B and Type $\mathbf{R}$ orientations. In the deformed state, however, the crystal grains of Type $\mathrm{B}$ and Type $\mathrm{R}$ orientations must be transformed to Type $\mathrm{Ca}$ and Type $\mathrm{Cb}$ orientations. As discussed previously, two types of crystal reorientation mechanisms resulting in Type $\mathrm{Ca}$ and $\mathrm{Cb}$ orientations must be localized at particular positions in the uniaxially deformed spherulites in such a way that Type $\mathrm{Ca}$ and $\mathrm{Type} \mathrm{Cb}$ are accentuated in the polar and equatorial zones, respectively. Type $\mathrm{Ca}$ orientation must also be accentuated in the particular zone of $\theta^{\prime}=45^{\circ}$, because it is in the maximum shear stress region, and can be designated as Type $\mathrm{Ca}_{45}$ in contrast to Type

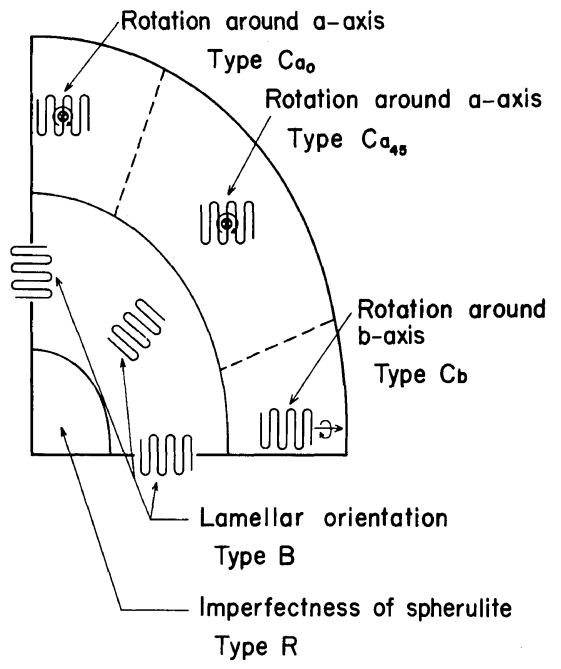

Figure 8. Schematic diagram demonstrating the four types of orientations of the crystal grains, Type $\mathrm{Ca}$, Type $\mathrm{Cb}$, Type B, and Type R orientations, within a uniaxially deformed spherulite.

$\mathrm{Ca}_{0}$ in the polar zone. Figure 8 is a schematic diagram demonstrating the respective types of orientations of the crystal grains within spherulite.

Fractional concentrations of the respective orientations of the crystal grains, $X_{i}\left(i=\mathrm{Ca}_{0}, \mathrm{Ca}_{45}, \mathrm{Cb}, \mathrm{B}\right.$, and $\mathrm{R}$ ), may be formulated, as a function of $\theta^{\prime}$, as follows:

(1)-1 Type $C a_{0}$ :

$$
X_{\dot{C}_{0}}\left(\theta^{\prime}\right)=f_{\mathrm{a}_{0}} \sigma \cos ^{2} \theta^{\prime}
$$

(1)-2 Type $\mathrm{Ca}_{45}$ :

$$
X_{\mathrm{Ca}_{45}}\left(\theta^{\prime}\right)=f_{\mathrm{a}_{45}} \sigma 4 \cos ^{2} \theta^{\prime} \sin ^{2} \theta^{\prime}
$$

(2) Type $C b$ :

$$
X_{\mathrm{Cb}}\left(\theta^{\prime}\right)=\left(1-f_{\mathrm{a}_{0}}-f_{\mathrm{a}_{4} 5}\right) \sigma \sin ^{2} \theta^{\prime}
$$

(3) Type B:

$X_{\mathrm{B}}\left(\theta^{\prime}\right)=f_{\mathrm{b}}\left[1-\left\{X_{\mathrm{Ca}_{0}}\left(\theta^{\prime}\right)+X_{\mathrm{Ca}_{45}}\left(\theta^{\prime}\right)+X_{\mathrm{Cb}}\left(\theta^{\prime}\right)\right\}\right]$

(4) Type R:

$$
\begin{aligned}
X_{\mathrm{R}}\left(\theta^{\prime}\right)= & \left(1-f_{\mathrm{b}}\right)\left[1-\left\{X_{\mathrm{Ca}_{0}}\left(\theta^{\prime}\right)\right.\right. \\
& \left.\left.+X_{\mathrm{Ca}_{45}}\left(\theta^{\prime}\right)+X_{\mathrm{Cb}}\left(\theta^{\prime}\right)\right\}\right]
\end{aligned}
$$

where $\sigma$ is a parameter representing the ease of

*2 For which the $u_{3}$ axis is taken as being parallel to the crystal $b$-axis (lamellar axis), instead of the $c$-axis, and the $u_{1}$ and $u_{2}$ axes as being parallel to the crystal $c$-axis (lamellar normal) and $a$-axis, respectively. 
transformation of the crystal grains from Type $\mathrm{B}$ and Type $\mathrm{R}$ orientations to Type $\mathrm{Ca}_{0}$, Type $\mathrm{Ca}_{45}$, and Type $\mathrm{Cb}$ orientations, and $f_{i}$ are fractions given by

$$
\begin{aligned}
& f_{\mathrm{b}}=\text { Type } \mathrm{B} /(\text { Type } \mathrm{B}+\text { Type } \mathrm{R}) \\
& f_{\mathrm{a}_{0}}=\text { Type } \mathrm{Ca}_{0} /\left(\text { Type } \mathrm{Ca}_{0}+\text { Type } \mathrm{Ca}_{45}\right. \\
& + \text { Type } \mathrm{Cb} \text { ) } \\
& f_{\mathrm{a}_{45}}=\text { Type } \mathrm{Ca}_{45} /\left(\text { Type } \mathrm{Ca}_{0}+\text { Type } \mathrm{Ca}_{45}\right. \\
& + \text { Type } \mathrm{Cb} \text { ) }
\end{aligned}
$$

under the conditions given by

$$
0 \leq f_{\mathrm{b}}, \quad f_{\mathrm{a}_{0}}, \quad f_{\mathrm{a}_{45}}, \quad \sigma \leq 1
$$

and

$$
\begin{aligned}
X_{\mathrm{Ca}_{0}}\left(\theta^{\prime}\right) & +X_{\mathrm{Ca}_{4} 5}\left(\theta^{\prime}\right) \\
& +X_{\mathrm{cb}}\left(\theta^{\prime}\right)+X_{\mathrm{B}}\left(\theta^{\prime}\right)+X_{\mathrm{R}}\left(\theta^{\prime}\right)=1
\end{aligned}
$$

By combining the orientation distribution function $P_{i}(\theta, \eta)$ with its fractional concentration of $X_{i}\left(\theta^{\prime}\right)$, the expansion coefficients of $A_{l 0 n}^{i}$ for the respective orientation distribution functions of the crystal grains are given by

$$
\begin{aligned}
A_{l 0 n}^{\mathrm{Ca}_{0}}= & \frac{1}{2 \pi} \int_{0}^{2 \pi} \int_{-1}^{1} P_{\mathrm{Ca}}(\theta, \eta) \Pi_{l}{ }^{n}(\xi) \cos (n \eta) \mathrm{d} \xi \mathrm{d} \eta \\
& \times \int_{-1}^{1} \frac{\left\{g\left(\theta^{\prime}\right)\right\}^{3}}{2} X_{\mathrm{Ca}_{0}}\left(\theta^{\prime}\right) \mathrm{d} \xi^{\prime}
\end{aligned}
$$

$$
A_{l 0 n}^{\mathrm{Ca}_{45}}=\frac{1}{2 \pi} \int_{0}^{2 \pi} \int_{-1}^{1} P_{\mathrm{Ca}}(\theta, \eta) \Pi_{l}{ }^{n}(\xi) \cos (n \eta) \mathrm{d} \xi \mathrm{d} \eta
$$$$
\times \int_{-1}^{1} \frac{\left\{g\left(\theta^{\prime}\right)\right\}^{3}}{2} X_{\mathrm{Ca}_{45}}\left(\theta^{\prime}\right) \mathrm{d} \xi^{\prime}
$$

$$
A_{l 0 n}^{\mathrm{Cb}}=\frac{1}{2 \pi} \int_{0}^{2 \pi} \int_{-1}^{1} P_{\mathrm{Cb}}(\theta, \eta) \Pi_{l}{ }^{n}(\xi) \cos (n \eta) \mathrm{d} \xi \mathrm{d} \eta
$$$$
\times \int_{-1}^{1} \frac{\left\{g\left(\theta^{\prime}\right)\right\}^{3}}{2} X_{\mathrm{Cb}}\left(\theta^{\prime}\right) \mathrm{d} \xi^{\prime}
$$

$$
\begin{aligned}
A_{l 0 n}^{\mathrm{B}^{\prime}}= & \frac{1}{2 \pi} \int_{0}^{2 \pi} \int_{-1}^{1} P_{\mathrm{B}}\left(\theta^{\prime}, \eta^{\prime}\right) X_{\mathrm{B}}\left(\theta^{\prime}\right) \Pi_{l}^{n}\left(\xi^{\prime}\right) \\
& \times \cos \left(n \eta^{\prime}\right) \mathrm{d} \xi^{\prime} \mathrm{d} \eta^{\prime} \\
= & \frac{1}{2 \pi} \int_{0}^{2 \pi} \int_{-1}^{1} P_{\mathrm{B}}\left(\theta^{\prime}, 0\right) X_{\mathrm{B}}\left(\theta^{\prime}\right) \Pi_{l}\left(\xi^{\prime}\right) \mathrm{d} \xi^{\prime} \mathrm{d} \eta^{\prime} \\
= & A_{l 00}^{\mathrm{B}^{\prime}}
\end{aligned}
$$

$$
\begin{aligned}
A_{l 0 n}^{\mathrm{R}}= & \frac{1}{2 \pi} \int_{0}^{2 \pi} \int_{-1}^{1} P_{\mathrm{R}}(\theta, \eta) \Pi_{l}{ }^{n}(\xi) \cos (n \eta) \mathrm{d} \xi \mathrm{d} \eta \\
& \times \int_{-1}^{1} X_{\mathrm{R}}\left(\theta^{\prime}\right) \mathrm{d} \xi^{\prime} \mathrm{d} \eta^{\prime} \\
= & \frac{1}{2 \pi} \int_{0}^{2 \pi} \int_{-1}^{1} P_{\mathrm{R}}(0,0) \Pi_{0}(\xi) \mathrm{d} \xi \mathrm{d} \eta \\
& \times \int_{-1}^{1} X_{\mathrm{R}}\left(\theta^{\prime}\right) \mathrm{d} \xi^{\prime} \mathrm{d} \eta^{\prime} \\
= & A_{000}^{\mathrm{R}}
\end{aligned}
$$

where $\xi^{\prime}=\cos \theta^{\prime}$.

Equation 43 formulating the expansion coefficient $A_{l 0 n}^{\mathrm{B}^{\prime}}$ for Type B orientation is based on a different fixation of the Cartesian coordinate 0$u_{1} u_{2} u_{3}$ to the crystal grain, as indicated in the previous footnote and in Figure 9 as well. Therefore, it is necessary to transform $A_{l 0 n}^{\mathrm{B}^{\prime}}$ to $A_{l 0 n}^{\mathrm{B}}$ by unifying the formulation with the others. This can be performed by modifying the relation given by eq 16 as follows:

$$
\begin{aligned}
\alpha_{l 0}^{j, \mathrm{~B}}= & 2 \pi\left(\frac{2}{2 l+1}\right)^{1 / 2}\left[A_{l 0 \mathrm{O}}^{\mathrm{B}^{\prime}} \Pi_{l}\left(\cos \Theta_{j}^{\prime}\right)\right. \\
& \left.+2 \sum_{n=2}^{l} A_{l 0 n}^{\mathrm{B}^{\prime}} \Pi_{l}{ }^{n}\left(\cos \Theta_{j}^{\prime}\right) \cos \left(n \Phi_{j}^{\prime}\right)\right] \\
= & 2 \pi\left(\frac{2}{2 l+1}\right)^{1 / 2} A_{l 00}^{\mathrm{B}^{\prime}} \Pi_{l}\left(\cos \Theta_{j}^{\prime}\right)
\end{aligned}
$$

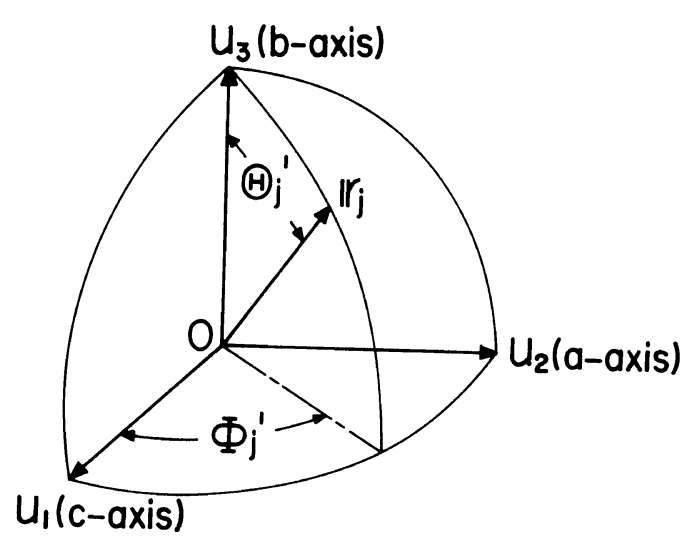

Figure 9. Polar and azimuthal angles, $\Theta_{j}{ }^{\prime}$ and $\Phi_{j}{ }^{\prime}$, specifying the orientation of the $r_{j}$ vector with respect to a Cartesian coordinate system $0-u_{1} u_{2} u_{3}$ fixed within a polyethylene crystal in a different way from that in Figure 1(b). 


$$
\begin{aligned}
= & 2 \pi\left(\frac{2}{2 l+1}\right)^{1 / 2}\left[A_{l 00}^{\mathrm{B}} \Pi_{l}\left(\cos \Theta_{j}\right)\right. \\
& +2 \sum_{n=2}^{l} A_{l 0 n}^{\mathrm{B}} \Pi_{l}{ }^{n}\left(\cos \Theta_{j}\right) \cos \left(n \Phi_{j}\right)
\end{aligned}
$$

where $\Theta_{j}^{\prime}$ and $\Phi_{j}^{\prime}$ are the polar and azimuthal angles specifying the orientation of the reciprocal lattice vector of the $j$-th crystal plane with respect to the Cartesian coordinate $0-u_{1} u_{2} u_{3}$ fixed within the crystal grain, as shown in Figure 9, in contrast to that in Figure 1(b). In practice, the transformation of $A_{l 0 n}^{\mathrm{B}^{\prime}}$ to $A_{l 0 n}^{\mathrm{B}}$ was carried out in the following way; calculating $A_{l 00}^{\mathrm{B}^{\prime}}$ from eq 28,33 , and 43, determining $\alpha_{l 0}^{j, \mathrm{~B}}$ from $A_{l 00}^{\mathrm{B}^{\prime}}$ from eq 45 for a given $j$, and further determining $A_{l 0 n}^{\mathrm{B}}$ from $\alpha_{l 0}^{j, \mathrm{~B}}$ by solving simultaneous equations of eq 46 with various $j$ up to 7 and $l$ up to 16.

The expansion coefficient $A_{l 0 n}$ for the orientation distribution function of the entire crystal grains can be obtained by averaging $A_{l 0 n}^{i}$ for the respective types of orientation; i.e.,

$$
A_{l 0 n}=A_{l 0 n}^{\mathrm{Ca}}+A_{l 0 n}^{\mathrm{Ca}_{45}}+A_{l 0 n}^{\mathrm{Cb}}+A_{l 0 n}^{\mathrm{B}}+A_{l 0 n}^{\mathrm{R}}
$$

Using $A_{l 0 n}$ thus averaged, the orientation distribution function of the crystal grains, $w(\xi, 0, \eta)$, can be determined from eq 12 with an approximation depending on the order of $l$. Similarly, the expansion coefficient $\alpha_{l 0}^{j}$ and further the orientation distribution function of the reciprocal lattice vector of the $j$-th crystal plane, $q_{j}\left(\zeta_{j}, 0\right)$, can be determined from eq 16 and 13 , respectively.

Figures 10 and 11 show the orientation distribution functions, $w(\xi, 0, \eta)$ and $q_{j}\left(\zeta_{j}, 0\right)$, thus calculated for the extension ratios of spherulite from 1.1 up to 1.4 by choosing the parameters so that the calculated results give the best fit to the observed orientation distribution functions in Figures 5 and 3. The values of the parameters chosen are listed in Table IV. As can be seen by a comparison of Figure
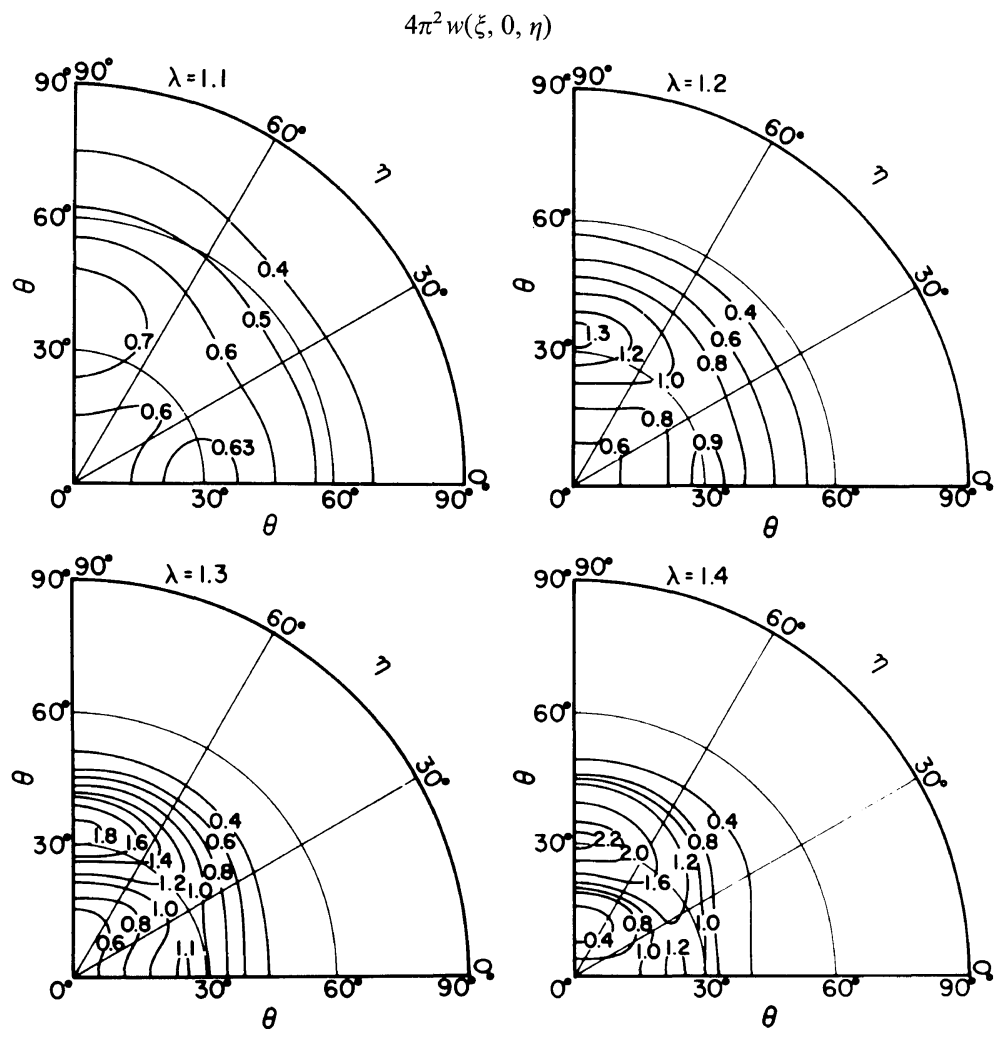

Figure 10. Change in uniaxial orientation distribution function of the crystal grains, $w(\xi, 0, \eta)$ with extension ratio of spherulite, calculated from spherulite deformation model using the model parameters listed in Table IV. 


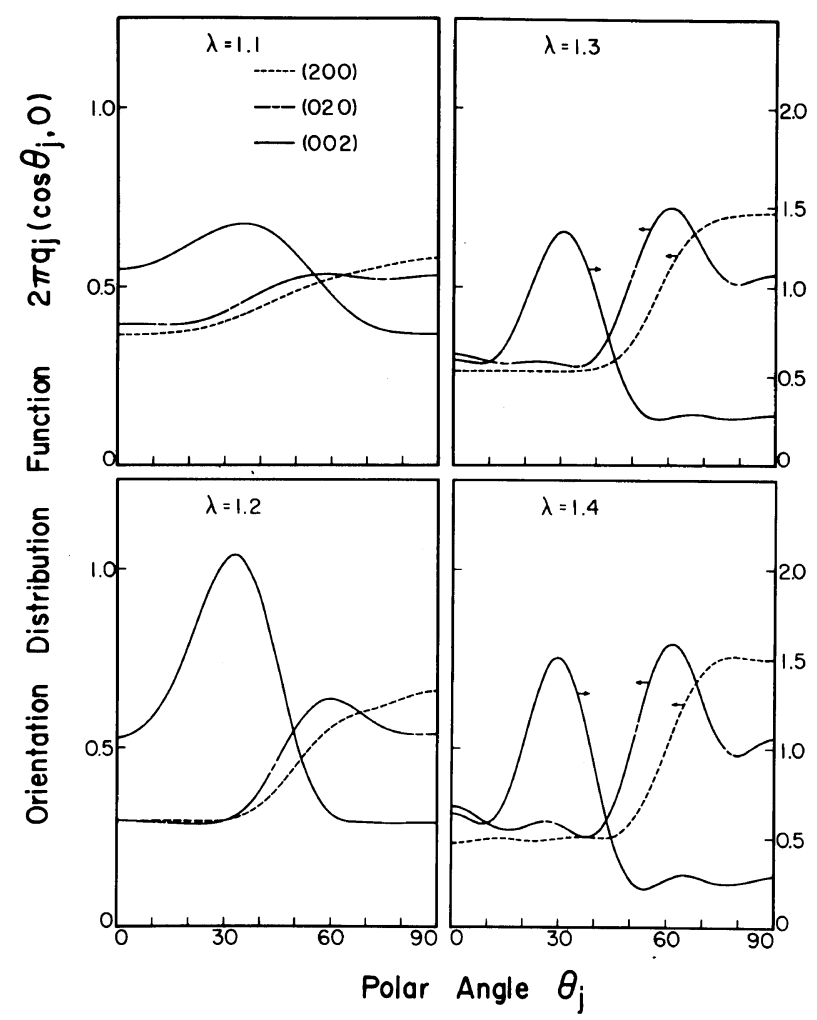

Figure 11. Changes in uniaxial orientation distribution functions of the principal crystallographic axes of polyethylene crystal, $q_{j}\left(\zeta_{j}, 0\right)$ with extension ratio of spherulite, calculated from the spherulite deformation model.

Table IV. Model parameters giving the best fit for the calculated orientation distribution function with that observed at various extension ratios

\begin{tabular}{ccccc}
\hline & \multicolumn{5}{c}{ Extension ratio } \\
\cline { 2 - 5 } Parameter & $\lambda=1.1$ & $\lambda=1.2$ & $\lambda=1.3$ & $\lambda=1.4$ \\
\cline { 2 - 5 } & 37.0 & 34.0 & 33.0 & 32.0 \\
$\theta_{0, \mathrm{Ca}}$ & 31.0 & 31.0 & 25.0 & 22.0 \\
$\theta_{0, \mathrm{Cb}}$ & 2 & 2 & 2 & 2 \\
$I_{\mathrm{a}}$ & 3 & 3 & 3 & 3 \\
$I_{\mathrm{b}}$ & 2 & 4 & 7 & 10 \\
$J_{\mathrm{a}}$ & 2 & 4 & 7 & 10 \\
$J_{\mathrm{b}}$ & 0.610 & 0.920 & 0.970 & 0.998 \\
$\sigma$ & 0.660 & 0.717 & 0.744 & 0.766 \\
$f_{\mathrm{a}}$ & 0.090 & 0.043 & 0.023 & 0.009 \\
$f_{\mathrm{a}_{45}}$ & 0.750 & 0.760 & 0.768 & 0.775 \\
$f_{\mathrm{a} 0}+f_{\mathrm{a}_{45}}$ & 0.970 & 0.900 & 0.710 & 0.598 \\
$f_{\mathrm{b}}$ & & & & \\
\hline
\end{tabular}

10 with Figure 5, the development of the two populations in the orientation distribution function is reproduced well by theory, with the more intensive population at $\theta$ a little larger than $30^{\circ}$ and $\eta$ of $90^{\circ}$ than the population at $\theta$ a little smaller than $30^{\circ}$ and $\eta$ of $0^{\circ}$. The orientation distribution functions, $q_{j}\left(\zeta_{j}, 0\right)$, calculated for the principal crystallographic axes (Figure 11) also agree fairly well with the observed functions (Figure 3), except for a small oscillation of intensity at the skirt portion. This oscillation must have originated from the adopted finite series of spherical harmoincs, and is especially true when the extension ratio is large and the intensity distribution becomes sharp.

The uniaxial deformation mechanism of spherulites will now be discussed in terms of the changes of the model parameters in Table IV with the extention ratio. As can be seen from the table, the parameter $f_{\mathrm{b}}$ which is the fraction of Type $\mathrm{B}$ orientation to Type B plus Type $\mathrm{R}$ orientations, 
continues to exceed $90 \%$ at relatively low degrees of extension, but decreases rather rapidly to $60 \%$ at relatively large degrees of extension. This suggests that the spherulitic crystalline texture is maintained almost perfect up to the extension ratio of 1.2 , beyond which the texture undergoes considerable disintegration.

The parameter $\sigma$, the ease of transformation of Type $\mathrm{B}$ and Type $\mathrm{R}$ orientations to Type $\mathrm{Ca}_{0}$, Type $\mathrm{Ca}_{45}$, and Type $\mathrm{Cb}$ orientations, increases and approaches unity with increasing extension ratio. The sum of $f_{\mathrm{a}_{0}}$ and $f_{\mathrm{a}_{4}}$, which is the fraction of Type $\mathrm{Ca}_{0}$ and Type $\mathrm{Ca}_{45}$ orientations to Type $\mathrm{Ca}_{0}$ plus Type $\mathrm{Ca}_{45}$ and Type $\mathrm{Cb}$ orientations, comes to almost $75 \%$ irrespective of the extension ratio. This suggests that the crystal reorientation mechanism involving the rotation about the crystal $a$-axis is much more predominant than that about the crystal $b$-axis at any extension ratio. The fraction $f_{\mathrm{a}_{0}}$ increases while the fraction $f_{\mathrm{a}_{45}}$ decreases, both with increasing extension ratio. The $f_{\mathrm{a}_{0}}$ is always much larger than the $f_{\mathrm{a}_{45}}$ at any extension ratio. Thus, the crystal rotation mechanism about the crystal $a$-axis seems to occur mostly in the polar zone but rarely in the $45^{\circ}$ region of the spherulite.

The parameters $I_{\mathrm{a}}$ and $I_{\mathrm{b}}$, which represent the sharpness of the orientation distributions of rotational angle of the crystal grains about their own $c$-axes for the $P_{\mathrm{Ca}}$ and $P_{\mathrm{Cb}}$, remain constants at 2 and 3 , respectively, irrespective of the extension ratio. On the other hand, the parameters $J_{\mathrm{a}}$ and $J_{\mathrm{b}}$ representing the sharpness of the distributions of orientation angle $\theta$ in the vicinity of $\theta_{0}$ for the $P_{\mathrm{Ca}}$ and $P_{\mathrm{Cb}}$, increase rather rapidly with an increase in the extension ratio. In other words, the orientation distribution functions, $P_{\mathrm{Ca}}$ and $P_{\mathrm{Cb}}$, become sharper in such a way that the $c$-axis orientation angle $\theta$ tends to be concentrated to $\theta_{0, \mathrm{Ca}}$ and $\theta_{0, \mathrm{Cb}}$, thus leading to two populations in the orientation distribution function $w(\xi, 0, \eta)$ at $\theta$ a little larger than $30^{\circ}$ and $\eta$ of $90^{\circ}$ and at $\theta$ a little smaller than $30^{\circ}$ and $\eta$ of $0^{\circ}$, respectively.

\section{CONCLUSION}

With a well-collimated high-flax beam of $\mathrm{Cu}-K \alpha$ line, the X-ray diffraction intensity distribution was observed for a high-density polyethylene film stretched uniaxially in $60^{\circ} \mathrm{C}$ warm water to various extension ratios up to 1.4. An extension rate as slow as about $1.5 \% / \mathrm{min}$ was adopted to avoid development of macroscopic necking.

The uniaxial orientation distribution function $q_{j}\left(\zeta_{j}, 0\right)$ was first investigated for 13 different $(j$-th) crystal planes including the (002) crystal plane, and then the orientation distribution function of crystallites (crystal grains) $w(\xi, 0, \eta)$ was determined at respective extension ratios, using a mathematical transformation procedure proposed by Roe and Krigbaum on the basis of the Legendre addition theorem.

It was found that two populations develop in the distribution function of $w(\xi, 0, \eta)$ at $\theta$ a little larger than $30^{\circ}$ and $\eta$ of $90^{\circ}$ and at $\theta$ a little smaller than $30^{\circ}$ and $\eta$ of $0^{\circ}$ and move to smaller $\theta$ to some extent, as the extension ratio is increased. The development of the former population seems to occur posteriorly but at much greater rate than the latter population.

The uniaxial deformation mechanism of polyethylene spherulites has been discussed quantitatively by comparing the dependence of $w(\xi, 0, \eta)$ upon the extension ratio with that calculated from a spherulite deformation model combining the crystal lamellar orientation in affine fashion with the reorientation of the crystal grains within the orienting lamellae.

The following conclusions have been drawn with regard to the spherulite deformation mechanism:

1) The spherulite deformation proceeds with its crystalline texture maintained almost nondisintegrated up to an extension ratio of 1.2 , beyond which the crystalline texture is considerably disintegrated, accompanied by the reorientation of the crystal grains within the lamellae.

2) The crystal reorientation mechanism can be represented in two ways which, though interacted and superposed with each other, involve rotations of the crystal grains about their own $a$ - and $b$-axes so as to orient their crystal $c$-axes toward the stretching direction. The rotations of the crystal grains about the crystal $a$ - and $b$-axes are surely associated, respectively, with lamellar shearing and lamellar detwisting and must be accentuated in the polar and equatorial zones of the uniaxially deformed spherulites.

3) The rotation of the crystal grains about their $a$-axes occurs posteriorly but at a much greater rate than that around their $b$-axes, resulting in a much more intensive population in the $w(\xi, 0, \eta)$ at $\theta$ a 


\section{K. Fujita et al.}

little larger than $30^{\circ}$ and $\eta$ of $90^{\circ}$ than the population at $\theta$ a little smaller than $30^{\circ}$ and $\eta$ of $0^{\circ}$ at later stages of extension up to 1.4 .

4) The populations move to smaller $\theta$ to some extent with increasing extension ratio, but never approach $0^{\circ}$ unless the extension ratio exceeds 1.4 , beyond which the macroscopic necking of the film specimen begins to appear and the spherulitic texture must be disintegrated into the so-called fiber texture of preferential $c$-axis orientation associated with the movement of the populations to the vicinity of $\theta \simeq 0^{\circ}$.

\section{REFERENCES}

1. H. D. Keith and F. J. Padden, J. Polym. Sci., 41, 525 (1959).

2. P. H. Geil, J. Polym. Sci., A-2, 3813 (1964).

3. Z. W. Wilchinsky, Polymer, 5, 271 (1964).

4. K. Sasaguri, S. Hoshino, and R. S. Stein, J. Appl. Phys., 35, 47 (1964).

5. K. Sasaguri, R. Yamada, and R. S. Stein, J. Appl. Phys., 35, 3188 (1964).

6. I. L. Hay and A. Keller, Kolloid Z. Z. Polym., 204, 43 (1965)

7. T. Oda, S. Nomura, and H. Kawai, J. Polym. Sci., A, 3, 1993 (1965).

8. T. Oda, N. Sakaguchi, and H. Kawai, J. Polym. Sci., C, 15, 223 (1966).

9. K. Kobayashi and T. Nagasawa, J. Polym. Sci., C, 15, 163 (1966).

10. R. S. Moore, J. Polym. Sci., A-2, 5, 711 (1967).

11. A. Keller and M. J. Machin, J. Macromol. Sci., Phys., B1, 41 (1967).

12. R. J. Samuels, J. Polym. Sci., C, 20, 253 (1967); J. Polym. Sci., A-2, 6, 1101 (1968).

13. R. G. Crystal and D. Hansen, J. Polym. Sci., A-2, 6, 981 (1968).

14. T. Oda, N. Sakaguchi, and H. Kawai, Kobunshi Kagaku, 25, 588 (1968).

15. T. Oda, M. Motegi, M. Moritani, and H. Kawai, Kobunshi Kagaku, 25, 639 (1968).

16. C. A. Garber and E. S. Clark, J. Macromol. Sci., Phys., B4, 499 (1970); Intern. J. Polym. Mater., 1, 31 (1971).

17. A. Peterlin, J. Mater. Sci., 6, 490 (1971).

18. S. Nomura, A. Asanuma, S. Suehiro, and H. Kawai, J. Polym. Sci., A-2, 9, 1991 (1971).

19. S. Nomura, M. Matsuo, and H. Kawai, J. Polym.
Sci., Polym. Phys. Ed., 10, 2489 (1972).

20. E. S. Clark, "Structure and Properties of Polymer Films," R. W. Lenz and R. S. Stein, Ed., Plenum Press, New York, 1973.

21. B. S. Sprague, J. Macromol. Sci., Phys., B8, 157 (1973).

22. T. Hashimoto, K. Nagatoshi, A. Todo, and $\mathbf{H}$. Kawai, Polymer, 17, 1063, 1075 (1976).

23. M. Matsuo, K. Hirota, K. Fujita, and H. Kawai, Macromolecules, 11, 1000 (1978).

24. K. Shimamura, S. Murakami, M. Tsuji, and K. Katayama, J. Soc. Rheol., Jpn., 7, 42 (1979).

25. T. Tagawa and K. Ogura, J. Polym. Sci., Polym. Phys. Ed., 18, 971 (1980).

26. T. Kyu, N. Yasuda, S. Suehiro, S. Nomura, and H. Kawai, Polym. J., 8, 565 (1976).

27. S. Suehiro, T. Yamada, H. Inagaki, T. Kyu, S. Nomura, and H. Kawai, J. Polym. Sci., Polym. Phys. Ed., 17, 763 (1979).

28. S. Suehiro, T. Kyu, K. Fujita, and H. Kawai, Polym. J., 11, 331 (1979).

29. S. Suehiro, T. Yamada, T. Kyu, K. Fujita, T. Hashimoto, and H. Kawai, Polym. Eng. \& Sci., 19, 929 (1979).

30. T. Kyu, N. Yasuda, S. Suehiro, T. Hashimoto, and H. Kawai, Polymer, 21, 1205 (1980).

31. T. Kyu, S. Suehiro, S. Nomura, and H. Kawai, $J$. Polym. Sci., Polym. Phys. Ed., 18, 951 (1980).

32. T. Kyu, S. Suehiro, and H. Kawai, Polym. J., 12, 251 (1980).

33. T. Kyu, M. Yamada, S. Suehiro, and H. Kawai, Polym. J., 12, 809 (1980).

34. S. Suehiro, T. Yamada, H. Inagaki, and H. Kawai, Polym. J., 10, 315 (1978).

35. T. Kyu, N. Yasuda, M. Tabushi, S. Nomura, and H. Kawai, Polym. J., 8, 565 (1976).

36. H. Kawai, T. Hashimoto, S. Suehiro, and T. Kyu, "The Strength and Stiffness of Polymers," R. S. Porter Ed., Marcel Dekker Inc., New York, in press.

37. H. Kawai, S. Suehiro, T. Kyu, and A. Shimomura, "Special Issue Commemorating the Tenth Anniversary of the Society of Rheology, Japan," Polymer Engineering Reviews, submitted to.

38. R. J. Roe and W. R. Krigbaum, J. Chem. Phys., 40, 2608 (1964).

39. W. R. Krigbaum and R. J. Roe, J. Chem. Phys., 41, 737 (1964).

40. R. J. Roe, J. Appl. Phys., 36, 2024 (1965).

41. S. Nomura, H. Kawai, I. Kimura, and M. Kagiyama, J. Polym. Sci., A-2, 8, 383 (1970). 